\title{
Verhandlungen der gynäkologischen Section der 51. Versammlung deutscher Naturforscher und Aerzte in Cassel.
}

I. Sitzung am 12. September 1878.

Vorsitzender: Herr Schwartz (Göttingen).

Schriftführer: Die Herren Fritsch (Halle), Pfannkuch (Cassel), Veit (Berlin).

1) Herr Leopold (Leipzig): Das skoliotisch und kyphoskoliotisch rachitische Becken; mit Demonstration von Präparaten und Abbildungen.

Im Laufe der letzten Jahre hatte ich mehrmals Gelegenheit, hochgradig kyphoskoliotische Frauen zu entbinden, welche nicht allein schon durch die enorme Difformität ihres Skelettes, sondern vor Allem dadurch, dass dieselbe rachitischen Ursprungs war, meine besondere Aufmerksamkeit auf sich zogen. Der Untersuchungsbefund und der eigenthümliche Verlauf ihrer Geburten liess mich eine der selteneren Formen des rachitischen Beckens annehmen, welche nur durch die mechanischen Verhältnisse der Kyphoskoliose bedingt sein konnten.

Es zeigte sich nämlich, dass bei diesen Frauen eine Art Trichterbecken mit auffallend verengtem Beckenausgang anzunehmen war; und zwar liess sich deutlich nachweisen, dass man ein stark rachitisches Becken vor sich hatte, jedoch mit mässig verengter Conjugata vera, man könnte eher sagen, mit einer relativ vergrösserten Conjugata vera, dagegen mit einem entschieden in allen Durchmessern verkleinerten, für rachitische Becken ungewöhnlich verengten Beckenausgange.

Mit anderen Worten: diese ganze Beckenconfiguration erinnerte lebhaft an das kyphotische Becken, wie es uns Breisky durch seine classischen Untersuchungen kennen gelehrt hat, und 
legte die Vermuthung nahe, dass das Becken dieser verkrüppelten Personen nur durch die Mechanik der ihnen anhaftenden Kyphoskoliose so wesentlich verändert war.

Diese Beobachtungen regten in mir das Studium der kyphoskoliotisch rachitischen Beckenform um so mehr an, als die praktisch hohe Bedeutung derselben sofort in die Augen sprang. In Folge dessen durchmusterte ich die Sammlungen des Leipziger Entbindungs- und pathologischen Institutes, und hatte dabei das Glück, unter einer grossen, halbvergessenen Skeletsammlung im pathologisch-anatomischen Institute einen höchst interessanten Fund zu machen; ich fand nämlich eine Reihe hochgradig skoliotisch und kyphoskoliotisch rachitischer Becken und ganzer Skelette, welche den folgenden Untersuchungen zu Grunde gelegt wurden. Ich habe die Präparate zeichnen lassen und eine Reihe der Originale Ihnen mitgebracht, und denke so am besten Ihnen die charakteristischsten Merkmale des durch Skoliose und Kyphoskoliose veränderten rachitischen Beckens vorführen zu können. Ausserdem fand ich noch in jener Sammlung durch Caries der Lendenwirbel entstandene kyphoskoliotische Becken; ein ebensolches ferner, ausserordentlich schönes, schrägverengtes aus dem Berliner pathologischen Institute hat mir Herr Geheimrath Virchow gütigst zur Verfügung gestellt. In Folge dessen kann ich dem rachitischen auch das cariöse kyphoskoliotische Becken zum Vergleich zur Seite stellen; somit liegt ein umfangreiches Material von 25 derartigen Becken resp. Skeletten vor, welche einen Einblick erlauben in die Genese, in das constante Verhalten und in die verschiedenen Modificationen jener Beckenform.

Wenn wir uns in der Literatur umsehen, so existirt meines Wissens keine einzige Arbeit, welche auf Grund eines grösseren Materiales ein genaues Bild entwürfe vom kyphoskoliotisch rachitischen Becken. Abgesehen von einzelnen zerstreuten Beschreibungen derartiger Becken, welche zu anderen Zwecken veröffentlicht wurden, so weist Schröder in seinem Lehrbuche ausdrücklich auf diese Beckenform hin und hebt besonders hervor, dass auf diese Modification des rachitischen Beckens bisher die Aufmerksamkeit noch nicht gelenkt worden ist. Unter ihm ist von König über diesen Gegenstand eine Arbeit erschienen, welche zwei Becken von lebenden Frauen und vier knöcherne Becken schildert. So sehr man nun diese Arbeit begrüssen muss, insofern als sie zuerst das grössere Augenmerk auf jene Becken gelenkt 
hat, so fehlt doch in ihr ein wichtiges Bindeglied, welches ich durch meine Mittheilungen zu ersetzen hoffe.

Da sich nämlich die Kyphoskoliose der Wirbelsäule aus zwei verschiedenen Abweichungen nach rückwärts und nach den Seiten zusammensetzt, und selbstverständlich jede dieser Abweichungen durch die Verhältnisse des Druckes und Gegendruckes ganz bestimmte Einflüsse auf ein Becken haben muss, so ist es für das Verständniss eines kyphoskoliotischen Beekens wohl unbedingt nothwendig, zunächst die Mechanik der einfachen Skoliose am rachitischen Becken kennen zu lernen, - und erst dann zu fragen: Wie wird ein skoliotisch rachitisches Becken durch eine hinzutretende Kyphose noch abgeändert?

Dieses wichtige Bindeglied, welches man in der Arbeit König's vermisst, hoffe ich Ihnen nun damit geben zu können, dass ich mein Material in zwei Gruppen theile, und Ihnen zunächst das skoliotisch rachitische Becken demonstrire.

Das rachitische Becken erfährt durch eine geringe oder stärkere Skolioseder Wirbelsäule keine Abschwächung seiner charakteristischen Eigenschaften. Im Gegentheil treten die Zeichen der Rachitis meistens sehr stark hervor, nur kommt hinzu, dass das Becken asymmetrisch wird, es kann selbst schrägverengt werden. Und zwar richtet sich diese Asymmetrie stets nach der Art der Skoliose. Bei starker rechtsseitiger Skoliose der Brustwirbel und compensirender linksseitiger der Lendenwirbel wird die linke Seite des Beckens zusammengedrängt, und umgekehrt. Zunächst muss aber hierbei festgehalten werden, dass diese Verhältnisse nur dann deutlich obwalten, wenn beide Unterextremitäten gebraucht werden und wenn die Skoliose schon in der Jugend entstanden ist.

Die Art des Zustandekommens geschieht auf folgende Weise: Die compensirende Lendenlordose zieht das Kreuzbein mit z. B. auf die linke Seite. Daher lastet auf ihr der grössere Druck von oben; und der grössere Gegendruck wird sie von unten treffen. In Folge dessen wird die linke Beckenseite zusammen, aber auch gleichzeitig nach aufwärts geschoben. In hohen Graden wird die linke Beckenhälfte, besonders im Beckeneingange, vollständig zusammengepresst, so dass sie für die Geburt ganz unbrauchbar ist.

Sehen wir nun hierbei, dass die Difformität des Beckens in der Hauptsache durch die Druckverhältnisse erzeugt wird, so 
der 51. Versammlung deutscher Naturforscher und Aerzte in Cassel. 451

treten aber eine Reihe anderer wichtiger Erscheinungen noch hinzu, welche auf das Klarste zeigen, wie der Zug der Muskeln, und ganz besonders der Bänder, das Becken ferner verändern. Zeigt sich der Muskelzug hauptsächlich an den breitgezogenen Tubera ischii, so erkennt man den bedeutenden Zug der zwischen Wirbelsäule und Darmbeinen verlaufenden Bänder sofort an der merkwürdigen Stellung der Darmbeine. Je stärker die Skoliose der Lendenwirbel ist, um so mehr wird das Darmbein der entgegengesetzten Seite gewissermassen nachgezogen, in Folge dessen nach der Mittellinie in die Länge und dabei die Schaufel nach einwärts gezogen. Daher haben die beiden Darmbeine einen ganz contrastirenden Stand; das auf der Seite der Lendenskoliosè ist kurz gedrungen, geradegestellt, nach aufwärts geschoben, das auf der anderen Seite breit und flach liegend, langgezogen und nach einwärts gerollt.

In den Becken, welche ich Ihnen vorlege, haben Sie durchweg eine der stärksten und einseitigen Verengerungen des Beckeneinganges. Die Conjugata vera bewegt sich zwischen $5^{1 / 2}$ und $71 / 2 \mathrm{Cm}$. Die Distantiae sacrocotyloideae differiren um $1-3 \mathrm{Cm}$; der Querdurchmesser des Beckeneinganges ist ziemlich normal. Beckenausgang ist weit und geräumig; nur in den Fällen von allgemeiner Verengerung des Beckens treten die Tubera ischii ziemlich aneinander, und ist der Schambogen auffallend spitz.

So sehen wir, dass die Skoliose das rachitische Becken vorwiegend im Beckeneingange verändert, und zwar die rachitischen Zeichen nicht nur nicht verwischt, sondern ihnen noch neue erschwerende Modificationen hinzufügt.

Am leichtesten dürfte sich die Entstehung dieses Beckens im erwachsenen Zustande verfolgen lassen, wenn Sie einen Vergleich anstellen zwischen dem Skelette des achtjährigen Mädchens $\left(\mathrm{S}_{4}\right)$ und den Skeletten $S_{1}, S_{2}$ und $S_{3}$.

Wie gestalten sich nun diese Verhältnisse, sobald sich zur Skoliose eine Kyphose der Wirbelsäule hinzugesellt?

Es erscheint wohl selbstrerständlich, dass eine so eingreifende Abweichung der Wirbelsäule, welche den Schwerpunkt des Körpers beträchtlich alterirt, auch die Configuration des Beckens mit beeinflusst. Doch thut sie es nur in bemerkbarerem Grade, um dies sofort vorauszuschicken, wenn die Kyphoskoliose in 
der Jugend aufgetreten ist und beide Unterextremitäten normaler Weise gebraucht werden; mit anderen Worten, wenn die Druckverhältnisse von oben und unten constante sind.

Gerade auf den letzteren Satz möchte ich das Hauptgewicht legen. Denn es wird sich zeigen, dass ein einseitiger Gebrauch eines Beines bei Kyphoskoliose des Thorax dem Becken sofort eine ganz andere Gestalt zu geben vermag.

Von wesentlichem Belange ist zunächst der Sitz, der Grad und die Gestaltung der Kyphoskoliose. Sie ist von grösstem Einflusse, wie dies schon Breisky für die rein kyphotischen Becken auseinandergesetzt hat, wenn sie in der Lenden- oder im unteren Theile der Brustwirbelsäule sich befindet, und zweitens, wenn die Kyphose sich einem spitzen Winkel nähert. Durch diese beiden Momente wird der Schwerpunkt des Körpers mehr nach rückwärts gelegt; daher muss ein ungewöhnlich starker Zug das Kreuzbein um seine Querachse drehen, wodurch das Promontorium nach rückwärts', die Kreuzbeinspitze nach vorwärts bewegt, und das ganze Kreuzbein gestreckt wird. Wenn wir aber festhalten, dass derartige Becken von Anfang an die rachitischen Zeichen darbieten, so werden offenbar diese hauptsächlichsten Charaktere gerade in die entgegengesetzten verwandelt. Demnach ist die Conjugata vera im Beckeneingange grösser, als bei den einfach skoliotischen Becken, das Kreuzbein ist mehr langgestreckt. Der ganze Beckeneingang ist mehr rund, und nimmt durch das Hereinspringen des Kreuzbeines und der Tubera ischii nach dem Beckenausgange hin in allen Durchmessern $a b$; somit entsteht eine Art Trichterform eines rachitischen Beckens.

Noch haben wir aber einen wichtigen Faktor vergessen für den Aufbau unserer Beckenform: es ist die Configuration, die Form der Kyphoskoliose. Sie ist es, welche dem eben geschilderten Becken noch eine wesentliche Veränderung aufdrücken kann, und welche davon abhängt, ob die Verbiegung der Wirbelsäule sich symmetrisch auf die linke wie rechte Seite des Körpers vertheilt, oder ob eine übermässige Ausbiegung auf einer Seite dieselbe mehr belastet.

Bei symmetrischen Verhältnissen braucht z. B. die Skoliose, selbst wenn sie sehr beträchtlich ist, keinen Einfluss auszuiuben, wie in Figur a 20; bei Asymmetrie dagegen wird dem Ueber- 
gewicht der Kyphoskoliose z. B. auf der linken Seite eine compensirende Skoliose der Lendenwirbel rechterseits die Wagschale halten, und in Folge dessen wird die rechte Beckenhälfte zusammengepresst sein, wie in Figur $T 79 ; a 38$.

Auf beifolgenden Tafeln habe ich das normale, das skoliotisch rachitische und das kyphoskoliotisch rachitische Becken in den verschiedenen Ebenen graphisch dargestellt. Es bedarf wohl keiner weiteren Andeutung, wie erheblich die Becken von einander abweichen. Auf zwei weiteren Tafeln habe ich das einfache kyphotische mit dem kyphoskoliotisch rachitischen Becken verglichen. Es erhellt sofort, dass das letztere ohne Zweifel dem ersteren etwas ähnelt; doch sind die rachitischen Charaktere immer noch so ausgeprägt, dass sie gar nicht übersehen werden können.

Des Interesses halber reihe ich hieran zwei Becken mit beträchtlicher Kyphoskoliose der Lenden- und Brustwirbel durch Caries, welche die eben angedeuteten Verhältnisse in noch stärkerem Grade zeigen, daher schon aus diesem Grunde nicht rachitischen Ursprunges sein können. Beide Becken sind deshalb hochinteressante Pendants, weil sie den bedeutenden Einfluss auf die weitere Gestaltung des Beckeneinganges bei verschiedenem Gebrauche der Unterextremitäten sehr schön nachweisen. An dem einen Präparate war rechtsseitige Coxitis mit Luxation, in früher Jugend entstanden; die rechte Hälfte des Beckens weit und atrophiseh. Das allein gebrauchte linke Bein hat den Beckeneingang durch Hereindrängen der Pfanne asymmetrisch gemacht. In dem anderen Präparate, das ebenfalls eine cariöse rechtsseitige Kyphoskoliose hat, und bei dem das Hauptgewicht anf die rechte Beckenhälfte gefallen ist, wurden beide Unterextremitäten gebraucht. Der hauptsächlichste Gegendruck von unten fand hier selbstredend vom rechten Beine statt, wodurch eine bedeutende rechtsseitige Verengerung mit schräger Verschiebung entstanden ist. Im Uebrigen zeigt gerade dieses Becken vorzüglich die Trichterform.

2) Herr A. Martin (Berlin): Zur Therapie der chronischen Metritis.

Redner charakterisirt kurz die verschiedenen Formen der Krankheit, bedingt durch Bindegewebshypertrophie mit Vergrösserung und Deformirung des Uterus, sei es noch unter seröser Durchtränkung oder bereits rorhandener Schrumpfung. Meist 
handle es sich nur um solche Formen, welche die Cervix anfallen resp. von ihr ausgehen (gestörte Involution des Uterus, acute in chronisch übergehende Metritis, nicht puerperale irritative Metritis). Gewöhnlich fände sich Ectropium, Erosionen, Geschwüre an der Vaginalportion, von denen Veit nachwies, dass sie Veränderungen darstellten, welche weit in die Muskulatur des Uterus sich erstreckten. Zuweilen beobachte man Geschwüre mit harten Rändern, entstanden durch Ausfall eines Gewebsstückes, carcinomähnliche Geschwüre bei chronischer Metritis der Cervix. Redner betont die Nutzlosigkeit der Localtherapie durch medicamentöse Aetzungen, Injectionen, Bepinselungen auf die Oberfläche. In Folge des geringen Erfolges dieser Verfahren sei die chronische Metritis in den Geruch der Unheilbarkeit gekommen. Das beste Mittel, das hypertrophische Gewebe zur Involution zu bringen, sei eine Schwangerschaft. C. Braun habe 1864 gezeigt, dass nach Abtragung der Cervix die Involution des ïbrigen Uterus bedeutender werde, als durch die Amputation der Cervix der Uterus verkürzt worden ist. Daher empfiehlt Martin, wo keine Schwangerschaft zu Stande komme, als Radikalmittel die Amputatio colli vorzunehmen. Martin trug so das Collum ab in frischen Fällen mit seröser Durchtränkung und in chronischen mit Induration. Er operirte $72 \mathrm{Mal}$; in einer Reihe von Fällen war anderweite lokale Behandlung vorhergegangen. Redner fand stets die Braun'sche Deduction bestätigt. Die Länge des Uterus war vor der Operation durchgängig vermehrt. Es wurden Stïcke abgetragen von $3-4 \mathrm{Cm}$. Neun Fälle boten vollständig das klinische Bild des Carcinoms der Portio, 64 reine Heilungen. In sieben Fällen erfolgten Nachblutung und Parametritis. Ein Fall starb an Typhus (Pyämie?). Die Nachbehandlung bestand in 7 bis 8 Tagen Bettlage; dann Entfernung der Nähte. Im Verlaufe mehrerer Wochen darauf völlige Involution.

Diese Rïckbildung des hypertrophischen Bindegewebes wird somit durch die Amputatio colli auf das Prompteste befördert.

Bezüglich der Methoden der Amputation stellt Martin die galvanokaustische Schlinge und das Ecrasement als nicht gut anwendbar hin, sondern zieht die Excision mit dem Messer vor, und zwar fand er die trichterförmige nach Hegar am brauchbarsten, weniger die von Simon und Kehrer. Mängel auch dieser Methode seien: die nicht immer zu vermeidenden Blutungen, die Störungen der Wundheilung durch weitergehende Erkrankung 
der 51. Versammlung deutscher Naturforscher und Aerzte in Cassel. 455

der Schleimhaut. Zur Vermeidung dessen näht Martin in folgender Weise, dass er dicht am Orificium externum je vorn und hinten zwei senkrechte Schleimhautfalten bildet, welche in die Scheide ragen und so rom Stumpfe fern gehalten werden. Im Uebrigen verfährt er nach Hegar. Nach dieser Modification habe er in 37 Fällen operirt.

Herr Kehrer (Giessen) stimmt Martin im Allgemeinen bei, verfähhrt aber nach seiner Methode in der Weise, dass er in die Cervix hinein vorn und hinten ein nach oben zugespitztes dreieckiges Stück excidirt und dann die quere Vereinigung vornimmt. Er habe so 40-50 Fälle mit Glück operirt. Er warnt vor Illusionen. Es kämen entzündliche Nachschübe vor.

Herr Schröder (Berlin) macht erst die Cervix durch künstliche Ectropiumbildung durch seitliche Einschnitte zugängig, lässt dann die Excision der Lippen folgen, in der Weise, dass er möglichst grosse Stücke der Vaginalschleimhaut der Portio sitzen lässt, und klappt dann diese Schleimhautlappen nach innen und oben in der Weise, dass der Cervixtrichter mit Vaginalschleimhaut ausgekleidet wird.

Herr 0lshausen (Halle) erklärt die Braun'sche Beobachtung anders. Die Schrumpfung und Verkleinerung des Uterus erfolge mehr durch die Nachbehandlung, Ruhe und Schonung, als durch die Amputation an sich. Ausserdem messe man das abgetragene Stück stets zu klein.

Herr Kehrer misst mit der Sonde vor und nach der Operation bis zur Commissur der Lippen, und glaubt die gerügte Täuschung für seine Fälle ausgeschlossen.

Herr $\mathrm{Ku}$ gelmann (Hannover) bezweifelt, dass die Indication zur Amputation so häufig zu stellen sei. Auch könne trotz derselben Endometritis fortbestehen und damit die Leucorrhoe. Der leichte Entschluss zu dieser Operation sei nicht berechtigt. Bei einem anderen Verfahren käme man auch zum Ziele. Er möchte warnen vor einem zu eifrigen Operiren.

Herr Martin replicirt, dass er wegen chronischer Metritis, nicht wegen Erosion und Endometritis operirt habe. Uebrigens habe er die Absonderung Seitens des Uterus stets beschränken können. Er weist den Vorwurf der leichtfertigen Indication ab, indem die Operation eine relativ kleine sei, mit der man gewiss noch vertrauter werde. Besser sei die Amputation, als monatelange vergebliche Heilungsbemühung. Durch Aetzungen u. s. w. 
können in einzelnen Fällen Heilungen vorkommen, aber gerade solche recidivirten gern.

Herr Kehrer stimmt bei, sofern die Amputation ein rasch zur Heilung führendes Verfahren ist.

Herr Schröder betont, dass es misslich sei, von den Indicationen zu sprechen. Er amputire gerade gern bei Schleimhanterkrankungen wegen der schweren Symptome. Die Operation selbst ist leicht und führt in kurzer Zeit zum Ziele. Gefährliche Neben- und Nacherkrankungen (Blutungen) seien selten. Nur eine Kranke verlor er an Tetanus. Er zieht eine vierzehntägige ungefährliche Behandlung einer längeren vor.

Herr $\mathrm{Kugelmann}$ betont, dass die Recidive die Resultate schmälern. Dann sei die Herabziehung des Uterus oft sehr schwierig, und steigere dieselbe die eventuellen Erkrankungen der Adnexa.

3) Herr Fritsch (Halle) zeigt ein schräges Becken. Dasselbe stammt von einem an Phthise gestorbenen achtzehnjährigen Mädchen, das an Epilepsie litt. In frühester Jugend ist der rechte Kreuzbeinflügel abgebrochen und hat sich nach hinten dislocirt. Hier ist er an der vorderen Fläche des Processus condyloideus festgewachsen. Dadurch hat das Kreuzbein rechts seinen Halt verloren und die rechte Seite des Körpers ist gesenkt und comprimirt. Das ganze Kreuzbein steht schräg, so dass die linke Incisura ischiadica enger ist als die rechte. Somit verhalten sich die Incisuren anders, als gewöhnlich beim schrägen Becken.

Die geringe Skoliose, welche durch die Compression der rechten Hälfte des Kreuzbeines entsteht, compensirt sich schon im vierten und fünften Lendenwirbel.

4) Herr Müller (Bern): Ueber Einwirkung des Pilocarpin a uf den Uterus.

Herr $M \ddot{i l l e r}$ stellte seine Versuche an puerperalen Uteris von Mehrgebärenden an und fand, dass die Contractionen, welche auf Pilocarpin allerdings eintreten, längere Pausen einhielten und nicht so ergiebig ausfielen, als bei Ergotin. Ausserdem wendete Herr Müller das Mittel drei Mal bei Beckenenge und ein Mal bei Hydrops mit Albuminurie zum Behufe der künstlichen Frühgeburt an mit negativem Erfolge. Herr Müller bezeichnet eine Discussion über den Werth des Mittels für die Geburtşhülfe als wünschenswerth. 
der 51. Versammlung deutscher Naturforscher und Aerzte in Cassel. 457

5) Herr Sänger (Leipzig): Weitere Vorschläge und Erfahrungen über das Pilocarpin in der Geburtshülfe. (S. dieses Archiv, Bd. XIV, Heft 1'.)

6) Herr Kehrer (Giessen) bespricht die Verlangsamung des Fötalpulses bei den Uteruscontractionen.

Die Thatsache erklärt sich weder durch die Theorie von Schwartz, weil eine einseitige Auspressung des Fruchtkuchens nicht wohl stattfindet, noch durch die Theorie von Schultze, weil 0-Mangel neben den pulsverlangsamenden Vaguskernen auch das Athmungscentrum erregt.

Ebensowenig lässt Herr Kehreír den gesteigerten allgemeinen Intrauterindruck als Ursache gelten. Die Versuche an lungenathmenden Geschöpfen in comprimirter Luft haben żwar Verlang-. samung von Puls und Athmung ergeben, beweisen aber nichts für den apnoëtischen, so zu sagen kiemenathmenden Fötus.

Redner hat mit Tritonenlarven experimentirt, so lange diese noch mit büschelförmigen äusseren Kiemen (den Analoga der Chorionzotten) athmen, und gefunden, dass selbst bei einem Wasserdrucke von 11 Mtr. die Häufigkeit des Herzschlages nicht geändert wird, unter der Voraussetzung gleicher Wassertemperatur vor und während des Druckversuches.

Diese Beobachtungen machen es wahrscheinlich, dass gesteigerter allgemeiner Inhaltsdruck bei der Wehe (nach Schatz nie höher als $1 / 3$ Atmosphäre) als Ursache der Pulsverlangsamung nicht zu betrachten sei. Dagegen kann partieller Hirndruck, welcher bei neugeborenen Thieren durch mässiges Herabdrücken der Scheitelbeine gegen den Schädelgrund bewirkt wird, den Puls verlangsamen, ohne, wie Schwartz an apnoëtischen Früchten zeigte, gleichzeitig Athembewegungen auszulösen. Besondere Versuche an neugeborenen Thieren haben ergeben, dass nach VagiSection die Hirncompression nicht mehr pulsverlangsamend wirkt.

Redner entwickélt an einem Schema den Satz, dass partielle Schädelcompression (mit Verdrängung der Cerebrospinalflüssigkeit gegen das Rückenmark) auch bei stehender Fruchtblase stattfinden könne, wenn der Schädel den drückenden Uteruswandungen anliegt und durch den ïbrigen Fötalkörper am Ausweichen gehindert ist. Er kommt zu dem Schlusse, dass mechanische Reizung der Vaguskerne den Fötalpuls bei den Wehen verlangsame. 
In der Discusion bestreitet Herr Lahs die Existenz eines Fruchtachsendruckes, giebt aber Formveränderung des Schädels bei den Contractionen zu.

Herr Kehrer will die Frage nach der Existenz eines Fruchtachsendruckes hier nicht erörtern; ihm genügt die zugegebene Veränderung der Schädelform bei den Wehen.

Herr O1shausen hält den allgemeinen Inhaltsdruck für ausreichend zur Erklärung der mechanischen Vagusreizung.

Herr Schwartz constatirt die Uebereinstimmung der Ansichten in Bezug darauf, dass die mechanische Reizung der Vaguskerne als Ursache der Pulsverlangsamung zu betrachten sei.

7) Herr Ruge (Berlin): Ueber Vaginitis granularis.

Er geht zunächst auf den histologischen Bau der normalen Scheidenschleimhaut ein, und erklärt dieselbe gegenüber den Untersuchungen von Preuschen's für drüsenlos. Demnach könne es sich bei der Vaginitis granularis, bei welcher er hauptsächlich verweilt, nicht um einen Ausgang der Granulabildung von Follikeln oder Drüsen aus handeln, vielmehr bestünden die entzündeten Wärzchen der Scheide aus einem stark gefässreichen Granulationsgewebe, dessen Decke bestünde aus den mit erhobenen und lediglich verschmälerten, resp. verschwundenen Epithelpapillen.

Die Rückbildung der Granula geschehe in der Weise, dass unter Abnahme der Hyperämie und Organisation der Zellen die Epithelpapillen ihre frühere Breite gegenüber den Gewebspapillen wieder einnehmen, bis das Niveau wieder hergestellt sei.

Redner verbreitet sich noch über die einfache Colpitis und die der Greisinnen and hebt hervor, wie selten die Erkrankungen wären, welche vom vaginalen, nicht vom cervicalen Theile der Portio vaginalis ausgingen.

Herr von Hoffmann (Wiesbaden) fand die Grenze des platten und Cylinderepithels erst am Hymen, nimmt also für die Scheide Auskleidung mit Cylinderepithel in Anspruch, und tritt auf die. Seite von Preuschen, welcher für die Vagina ein modificirtes Cylinderepithel aufstellt. Beim Fötus herrsche unter den Zellen im geschichteten Vaginalepithel die Birnform vor.

Herr Leopold fand in den Scheiden erwachsener Personen niemals Drüsen. Die Granula seien oft epithellos und blu- 
teten leicht, wie es Redner für die excoriirten Vaginitiden der Greisinnen geschildert hat.

Herr Ruge möchte die Angaben von Preuschen insofern in Schutz nehmen, als bei Vaginen, die durch Pruritus verändert sind, Bilder vorkommen, die denen Preuschen's ähnlich sind. Betreffs des Cylinderepithels warnt Redner, nicht ganz frische Präparate zur Untersuchung zu nehmen.

8) Herr Veit (Berlin): Demonstration eines Präparates von doppelseitiger Achsendrehung der Ovarien.

Bei 94, von Schröder ausgeführten Ovariotomien, wurde 13 Mal Achsendrehung eines Eierstockes beobachtet. Das Präparat stammt von einer 29jährigen Frau, die drei Mal geboren hatte und eine Zeit lang unter der Diagnose einer Parametritis sinistra behandelt worden war. Drei Monate nach der letzten Entbindung erkrankte sie an einer heftigen Peritonitis, welche als die Folge einer Achsendrehung eines Ovarientumors angesehen wurde. Bei der Ovariotomie bestätigte sich dieselbe. Ausserdem fand sich linkerseits das Ovarium ebenfalls durch Achsendrehung abgeschnürt.

Herr Olshausen hält die Diagnose der Torsion für nicht so schwer, und stellt unter den Symptomen in erste Linie die Peritonitis.

Herr Breisky (Prag) fragt, ob nicht aus der Beschaffenheit der durch Probepunction entnommenen Flüssigkeit ein Schluss gemacht werden könne auf stattgefundene Torsion. Es sei ihm ein Fall begegnet, wo nach einer ersten Punction bei einer Frau, deren Unterleib durch Kystom stark ausgedehnt war, die herausgeholte Flüssigkeit aashaften Geruch besass. Bei der Ovariotomie fand sich Achsondrehung.

Herr Freund führt einen ähnlichen Befund an und fügt Bemerkungen bei über den Mechanismus der Torsionen. Er habe beobachtet, dass bei Vorhandensein von Torsionen. der Ovarientumor stets an irgend einer peripheren Stelle (Darm, Netz, Bauchwand) festgeheftet sei. Es fänden sich auch Torsionen an den Adhäsionssträngen selbst, analog denen am Ligamentum latum, dem Uterusstiel, z. B. am Netz. Das Ovarium sei gewissermassen zwischen zwei Fäden aufgehängt; Czerny habe Freund gegenüber die Vermuthung geäussert, dass möglicherweise. schon in der Fötalperiode das Ovarium peripher verwachsen sein könne, namentlich mit dem Netze. Ueberhaupt finde sich eine halbe AchsenArchiv f. Gynäkologie. Bd. XIII, Hft. 3. 
drehung des Stieles bei jeder Ovariengeschwulst. Der Tumor ovarii steigt erst nach oben, dann aber unter partieller Torsion nach vorn, weshalb die Tube hinter und unter ihm zu liegen pflegt. Uebrigens gesteht Freund zu, dass auch andere Mechanismen stattfinden können.

Herr Veit führt zwei Fälle an, wo keine Adhäsion und doch Torsion bestand.

Auch Herr Olshausen sagt aus, dass in der Mehrzahl der Fälle die Tumoren nicht adhärent wären. Er glaubt, dass freie und kleinere Tumoren sich leichter torquiren können.

Herr Winckel replicirt auf Herrn Breis ky's vorherige Anfrage. Er beobachtete ebenfalls an einer nicht fiebernden Patientin auf Probepunction eine stinkende Flüssigkeit mit reichlichen Bacterien, deren Eindringen nur auf den Blutwegen geschehen sein konnte. Die Diagnose wurde nicht gestellt. Jedenfalls war es kein Ovarientumor.

Herr Martin schildert einen Fall von Ovariotomie bei 1/2/2maliger Torsion wegen drohender Symptome. Adhäsionen fanden sich nicht.

Herr de la Camp (Hamburg) warnt, aus der fauligen Beschaffenheit von Punctionsflüssigkeit auf Ovarientumoren mit Achsendrehung zu schliessen. Er habe ein Mal wegen vermeintlichen Tumors, welcher ebenfalls stinkende Flüssigkeit enthielt, die Laparotomie gemacht und es höchst wahrscheinlich mit Parametritis zu thun gehabt.

Herr Freund hält seine Ansicht Herrn Martin gegenüber aufrecht. Bei einer Verwachsung eines Ovarientumors mit dem Netze brauche das Netz nicht gespannt zu sein. Ohne doppelte Befestigung könne eine dauernde Drehung kaum stattfinden.

Herr Fritsch hebt hervor, dass der frei bewegliche, kaum tixirte Fötus sich doch auch drehe.

9) Herr Runge (Strassburg): Ueber den Einfluss der Herabsetzung des Blutdruckes auf das Leben der Frucht.

Anknüpfend an frühere Untersuchungen über den beregten Gegenstand bemerkt Redner, dass der Tod der. Jungen bei Alkalientziehungen des Mutterblutes sich nicht durch diese an sich erkläre. Es träte anch ein Absterben der Jungen ein, wenn man mittels Durchtrennung des Halsmarkes ein rasches und bedeuten- 
des Absinken des Blutdruckes erzeuge. Mittel, welche diesen herabsetzten, müssten somit das Kind gefährden, z. B. das Chloroform. Kann màn dem Mutterthiere so viel Chloroform geben, dass die Jungen sterben, die Mutter aber nicht, so ist dieser Satz bewiesen, was ihm auch wirklich gelang. Liess man das Chloroform nach einiger Zeit der Narkose weg, so erholten sich die Jungen nicht wieder. Auch Aethernarkose bringt diese zum Absterben, jedoch langsam.

10) Herr Zweifel (Erlangen): Ueber die Grundsätze der Abnabelung.

Gestützt auf Blutbestimmungen in der Placenta hatte Redner ${ }^{1}$ ) den Vorschlag gemacht, die Abnabelung in Zukunft anders auszuführen und allmälich auch die Hebammen anders zu lehren, als dies bisher geschehen ist. Das Resultat der Blutbestimmungen nach Welker's Methode ergab für die gewöhnliche Abnabelung einen Blutgehalt von $192 \mathrm{Gr}$, bei der sogenannten Spätabnabelung nur 92 Gr. Bei diesem letzteren Verfahren wurde zuweilen abgewartet, bis die Placenta durch den Credé'schen Handgriff ausgepresst, dann die Nachgeburt noch zwischen beiden Händen möglichst blutleer gedrückt und das Blut des Nabelstranges gegen das Kind hin ausgestreift war. Erst nach dieser Entleerung der Placenta wurde der Nabelstrang unterbunden. Der Blutgewinn des Kindes würde danach etwa $100 \mathrm{Gr}$. betragen und diese Zahl stimmt ganz überein mit dem Quantum, das Schücking als Reserveblut bezeichnet hatte.

Gegen das Resultat dieser Blutbestimmungen hat nun L. Me ye r Opposition gemacht, indem er bei mehreren Versuchen nur einen Unterschied von circa $16 \mathrm{Gr}$. gefunden. Es wäre bei einem so geringen Unterschied fraglich, ob sich dabei die Unbequemlichkeit der Spätabnabelung noch mit in den Kauf nehmen liesse. Um die Frage einer Entscheidung näher zu bringen, wurden in der Klinik in Erlangen die Versuche weiter fortgesetzt. Die Ergebnisse waren hier: 178,5 Gm. Blutgehalt der Placenta bei gewöhnlicher Abnabelung, 97,5 Gm. bei der Spätabnabelung, also ein Unterschied von $81 \mathrm{Gm}$. Das ist eine entschiedene Bestätigung der erst gemachten Publication, und hält Redner darum auch an den daraus gezogenen Consequenzen fest.

1) Centralblatt für Gynälologie $1878, \mathrm{Nr} .1$. 
11) Herr Zweifel: Die innere Athmung im Blute der Placenta.

Wenn einem Hunde die Carotis an zwei Stellen unterbunden wird, so dunkelt das abgeschlossene Blut in ca. einer Viertelstunde ab. Der Sauerstoff verschwindet vollkommen. Darin waren alle Autoren einig, aber es entstand bald eine Controverse darüber, wohin der Sauerstoff gehe. Der oben erwähnte Fundamentalversuch ist ziemlich gleichzeitig von Pflüger, von HoppeSeyler und von Schmidt gemacht worden, ohme dass der eine ihn vom andern kannte. Hoppe-Seyler machte noch eine Modification des Versuches, aus welcher er schloss, dass die Hauptzehrung des Sauerstoffes von Seiten der Gefässwand stattfinde.

Im Nabelstrange machte sich nun die auffallende Thatsache geltend, dass das Blut, welches bei der Spätabnabelung in den Arterien hellroth geworden war, viel länger, bisweilen stundenlang die rothe Farbe behielt. Dies geschah auch dann, wenn der Strang rom Luftzutritt and einem entsprechenden Gasaustausch durch Wasser abgeschlossen war. Zur Erklärung dieses Befundes müsste angenommen werden, dass sich entweder das Blut des Fötus anders verhalte, als dasjenige des Erwachsenen, oder die Wände der Nabelgefässe weniger Sauerstoff zehren, als dies in Körperarterien der Fall ist.

Für die letztere Auffassung würde es sich dann wiederum fragen, ob die Nabelarterien wegen ihrer eigenen Beschaffenheit (da sie keine Vasa vasorum besitzen), oder weil sie keinen Sauerstoff an das umliegende Gewebe abgeben können, weniger Sauerstoffzehrung haben. Es ist kaum wahrscheinlich, dass sich das Blut anders verhält, dann muss das differente Verhalten aus den Verhältnissen der Nabelgefässe erklärt werden.

Wissenschaftlich von Werth wäre dabei, dass beim Fötus für eine möglichst gute Conservirung des Sauerstoffes gesorgt wäre, indem von der Placenta bis zum Kinde Gefässe eingeschaltet wären, welche ungewöhnlich wenig Sauerstoff verbrauchten.

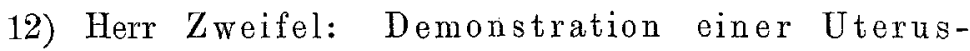
douche zum Ersatz der gewöhnlichen Wandirrigateure.

Weil die Klystierspritzen schwer und plump sind, in ungeschickten Händen schon grossen Schaden angerichtet haben und meistens, wenn man sie brauchen will, nicht dienen, hat man vielfach die Irrigateure zum Ersatz der Klystierspritzen eingeführt. 
der 51. Versammlung deutscher Naturforscher und Aerzte in Cassel. 463

Aber diese Instrumente sind schwer zu transportiren, und darum z. B. für die private Praxis nicht so gut verwendbar. Um diesen letzteren Vortheil auch zu erreichen, liess Rednex an den gewöhnlichen Uterusdonchen einen Saugballon construiren.

Dieser Saugheberirrigateur besteht danach aus dem gewöhnlichen Fusse von Zink, einem 2 Mtr. langen Kautschukrohr; am entgegengesetzten Ende befindet sich ein birnförmiger Saugballon, an welchen ein Mutterrohr von Zink angeschraubt werden kann. Dieses letztere ist also isolirt zu desinficiren.

Es ist noch ein Vortheil an diesem Apparate zu erwähnen, dass nämlich dabei nicht wohl Luft in die Genitalien eingespritzt werden kann. Es muss der Finger so lange die Oeffnung des Ausflussrohres verschliessen, bis sich der Saugballon und das ganze Rohr mit Wasser angefiullt hat.

Wo man am Ausflusse mehrere Oeffnungen haben will, ist ein Hahn nothwendig. Diese Apparate sind für die Hebammenschülerinnen in Erlangen bereits zum. Ersatz der Klystierspritzen eingeführt worden, und sind zu beziehen von dem dortigen Instrumentenmacher Kleinknecht.

13) Herr Zweifel: Demonstration eines Saugfläschchens mit Einrichtung zum Selbstabsaugen.

Aus einem recht kleinen Saugfläschchen mit der gewöhnlichen Einrichtung kann sofort ein Selbstabsauger gemacht werden durch Aufsetzen eines anderen Korkes, der doppelt durchbohrt ist, wovon ein Glasröhrchen mit einem gläsernen Brustwarzenhütchen, das andere mit einem Glasstïck in Verbindung steht, das die Mutter in den Mund nimmt, um selbst die Milch aus der Brust zu ziehen. Es ist nur darauf zu sehen, dass die Mündungen der beiden Glasröhren im Fläschchen nicht im gleichen Niveau stehen, weil 
sonst leicht die Milch aus der einen Röhre in das Saugrohr übertritt und der Mutter direct in den Mund fliesst.

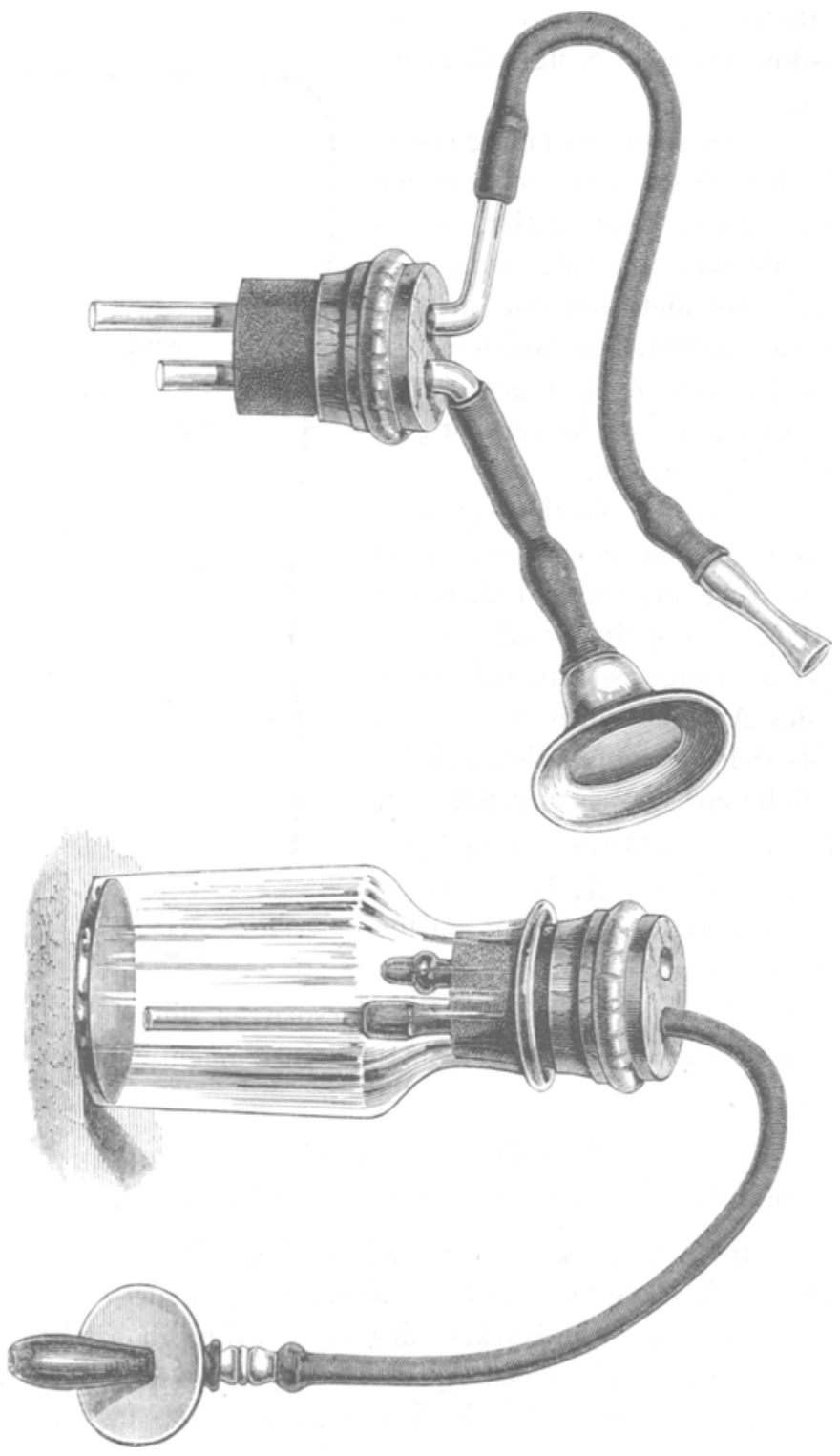

Anwendung kann dieses Fläschchen ganz besonders dann finden, wenn das Kind die Brustwarzen nicht fassen kanrı. Als Be- 
der 51. Versammlung deutscher Naturforscher und Aerzte in Cassel. 465

weis für die Brauchbarkeit kann Verf. erwähnen, dass eine Mutter monatelang ihr Kind ausschliesslich so ernährt hat. tistik.

14) Herr Dohrn (Marburg): Zur puerperalen Sta-

Herr Dohrn bezieht sich auf den Abschnitt 5-7 der Arbeit von Herrn Böhr über die Häufigkeit des Todes im Wochenbette in Preussen, in der Zeitschrift für Geburtshülfe und Gynäkologie, III, 1, worin die Provinz Hessen-Nassau als diejenige Provinz Preussens hingestellt worden ist, welche die höchste Mortalitätszahl aufzuweisen habe. Herr Dohrn fühle sich veranlasst, den Fehler aufzudecken, welchen Böhr mit dieser Verunglimpfung gemacht habe. In Nassau sei es mit der puerperalen Statistik wohl eben so gut bestellt, wie in Posen und Schlesien. Seit 1866 habe sich die Geburtsstatistik in HessenNassau relativ verschlechtert, da die Verpflichtung dazu aufgehört habe. Von 1868 bis 1874 sei ron 9\% der im Wochenbett gestorbenen Frauen die Todesursache nicht angegeben, und doch würden in Hessen-Nassau mehr Fälle gebucht, als in dem weitläufigen Posen und Holstein. In Hessen-Nassau käme eine Hebamme auf 22, in Posen eine auf 122 Wöchnerinnen. Es hänge von der Buchführung, nicht vom geburtshülflichen Personale ab, wie die Resuitate ausfielen, und weil die Buchführung nicht genau genug verfahren, so sei entschieden die Zahl der puerperal Todten eine grössere, als sie jeden Orts gebucht werde. Für Hessen-Nassau und für die ganze Monarchie sei es wünschenswerth, dass die früheren statistischen Einrichtungen der erstgenannten Provinz wiederhergestellt respective wieder eingerichtet würden.

Herr Schröder sieht eher in der geringen Zahl der Wöchnerinnen eine Möglichkeit für Verschleppung des Puerperalfiebers, und nimmt $\mathrm{Böhr}$ in Schutz, als habe er polemisch gegen HessenNassau vorgehen wollen. Böhr habe es sich nicht erklären können, weshalb die Procentziffer für Hessen-Nassau eine so hohe war, und habe einfach die vermeintliche Thatsache hingestellt.

Herr D o h rn erklärt, dass mit der Veröffentlichung der Zahlen hätte vorsichtiger verfahren werden müssen. Man hätte bei ihm anzufragen gehabt. Er hätte dann den Fehler aufklären können. Die Tendenz, überall Verschulden zu suchen, sei zu verurtheilen.

Zuletzt wendet sich Herr Dohr $n$ gegen einen ihm sehr anrüchig erscheinenden Passus des Aufsatzes der Berliner Puerperalcom- 
mission, nämlich über den Appell an die Ehemänner: Sie sollten nicht zulassen, dass die Aerzte und Hebammen untersuchten, ehe sie sich desinficirt hätten. Ein solches Ansinnen sei so lange energisch zurückzuweisen, als nicht der Appell an die Aerzte erschöpft sei. (Lebhaftes Bravo!)

Herr Schröder meint, es solle doch wohl keine Kritik der Böhr'schen Arbeit gegeben werden.

Herr Dohrn antwortet, dass er sich allerdings für dazu berechtigt halte.

Herr Martin erklärt, dass die Arbeiten der Puerperalcommission in Berlin der Böhr'schen Arbeit fern gestanden hätten und dass die Commission sich gegen das statistische Material misstrauisch verhalten habe. Es wurde eine Sichtung desselben vorgenommen und die gefundenen Zahlen als nur annähernde hingestellt. Die Berliner Commission treffe darum der Vorwurf Dohrn's nicht.

Herr Winckel warnt vor weiterer Ausspinnung der Discussion. Es müsse, sagt er im Sinne Dohrn's, zunächst darüber eine Einigung erzielt werden, was die Basen einer rationellen Statistik sein sollen. Heute möge nur eine Anregung dazu gegeben sein. Verhandlungen könnten noch nicht stattfinden.

15) Herr de la Camp (Hamburg): Fall von Uterusfibrom mit Exstirpation.

Frl. B., 28 Jahre alt, kam im Anfange des Jahres 1871 wegen eines Tumor im Leibe in Behandlung. Der Tumor hatte die Grösse eines fünfmonatlichen Uterus, war ziemlich weich, lag in der. Mittellinie über der Symphyse, dabei waren die Menses regelmässig, sparsam. Patientin nicht deflorirt, weshalb eine Untersuchung per raginam wenig über die Natur constatiren konnte. Im. Juli desselben Jahres während der Menses bekam Patientin plötzlich Schmerzen, und es gingen eine Menge. Hydatiden mit fadenartigen velamentösen Massen während mehrerer Tage ab. Hierauf verlor sich der Tumor. Patientin erholte sich, und erfreute sich während mehrerer Jahre einer guten Gesundheit und regelmässiger menstrueller Functionen. Der Uterus war im October deśselben Jahres von normaler Grösse, Orificium, so weit man fühlen konnte, normal.

Im März 1874 meldete sich Patientin abermals wegen eines Tumor im Leibe, der ohne Schmerz entstanden, mehr nach rechts 
der 51. Versammlung dentscher Naturforscher und Aerzte in Cassel. 467

am, event. im Uterus seinen Sitz hatte, consistenter war, und für einen Tumor ovarii angesprochen werden konnte, Allgemeinbetinden dabei gut, Menses regelmässig aber stark.

Im Jahre 1875 zeigten sich nach rechts oben und in der linken Bauchhöhle weitere Geschwülste, deren solide Beschaffenheit die Diagnose des Tumor ovarii wankend machten, und eine Anfang 1876 in der Chloroformnarkose vorgenommene Untersuchung bestätigte den Zusammenhang mit dem Uterus, in den mit Mühe die Sonde eingeführt werden konnte und eine bedeutende Verlängerung der Höhle zeigte. Herr Prof. Hegar, der die Gefälligkeit hatte, bei Gelegenheit dieser Versammlung der Aerzte und Naturforscher in Hamburg die Kranke. zu untersuchen, bestätigte meine Diagnose, und rieth zu der, wenn gleich schwierigen Operation. Die Sache zog sich dennoch bis zum Frühjahr 1878 hin, nur Soolbäder und Evacuantia verschafften der sehr belästigten Kranken einige Erleichterng, und sie wurde nun ins Hamburger Freimaurer-Krankenhaus aufgenommen.

Nach nochmaliger gründlicher Untersuchung wurde sie am 8. Mai der Operation unterzogen; dieselbe wurde von Herrn Dr. Wolf, der die chirurgischen Functionen an genanntem Hospital besorgt, mit grosser Unsicht und gutem Erfolge ausgeführt. Die Technik der Operation war wenig von der Exstirpation der Ovarialtumoren verschieden, natïrlich unter vollständiger Desinfection aller helfenden Hände und Instrumente, und unter Carbolspray. Bemerkenswerth war, dass der Bauchschnitt von der Symphyse bis zum Scrobiculus cordis verlängert werden musste, und dass dennoch die Drehung und Herausbeförderung des Tumor viel Mühe machte; or betrug den zehnten Theil des Körpergewichts der Patientin, 11 Pfund gegen 110 Pfund. Der ziemlich verlängerte Uterus wurde dann schräg in eine Wells'sche Klammer gelegt, so dass das Ovarium der rechten Seite mit weggenommen wurde, das linke sitzen blieb. Es bildete sich auf diese Weise ein ganz guter Stiel, der unterhalb der Klammer noch mit einer Drahtecraseurschlinge umschnürt, dann abgeschnitten und noch mit dem Glüheisen verschorft wurde. Eine stärkere Blutung aus dem Plexus pampiniformis érforderte noch besondere Unterbindungen und Toilette. Die Bauchnaht wurde mit 23 Suturen geschlossen, und dann Salicylwatteverband angelegt. Der Verlauf war ein recht günstiger, so dass die Patientin schon in der neunten Woche eine Reise nach L. machen konnte. 
In den ersten Tagen trat grosse Depression der Kräfte ein; Temperatur 36, Puls 60, so dass Cognac, Eis, Aetherinjectionen subcutan gemacht werden mussten, am zweiten Tage hob sich Puls und Temperatur, und es trat etwas Schlaf ein. Urin musste entfernt werden. Am dritten Tage nahm die Kranke Haferschleim, und Flatus gingen ab, dennoch blieb grosse Beklemmung und wurde ein elastisches Darmrohr eingeführt, was durch den Abgang der Winde grosse Erleichterung gewährte.

Vom vierten Tage erhielt sich die Temperatur auf 37 bis 38,5 und stieg nur zwei Mal durch Zwischenfälle, so am fünften. Tage wegen etwas Durchfall, der sich aber wieder verlor. Der Verband wurde am dritten Tage zuerst gewechselt. Am sechsten Tage verlangte die Kranke mehr Nahrung, an siebenten Tage konnten schon einige Nähte entfernt werden, und am 15. Tage die Klammer. Am 16. Tage zeigte die Wunde sich trocken, die Temperatur stieg auf 40, so dass Erysipel befürchtet wurde, indess auch dies verlor sich in den nächsten Tagen nach einigen Dosen Chinin, und nun ging die Heilung ohne Zwischenfall von statten, so dass die Kranke zwei Monate nach der Operation entlassen werden konnte. (Der exstirpirte Tumor wird vorgelegt.)

16) Herr Schröder: Ueber Laparotomie bei Uterusmyomen.

Herr Schröder will hauptsächlich eine Discussion angeregt haben, welche Methode wohl die vorzüglichste sei, als welche er die Péan'sche mit gewissen von ihm angegebenen Modificationen bezeichnet. Er macht die keilförmige Excision, und zwar in der Weise, dass er zunächst durch die Gegend des inneren Muttermundes eine Nadel durchführt und dann den Uterus nach beiden Seiten abschnürt. Dann schneidet er den Tumor oberhalb der Ligatur so heraus, wie ein Stück aus einer Melone, näht dann beide Lappen zusammen und versenkt. Von sechs so operirten Frauen starb nur eine.

Herr P. Müller (Bern) hat in zwei Fällen den Stielrest mit der Spencer Wells'schen Klammer fixirt, und zwar um gleichzeitig den bestehenden Vorfall zu beseitigen.

Herr Winckel hat zwei Fälle in einer dem Schröder'schen Verfahren ganz ähnlichen Weise operirt, will sich aber nicht die Priorität zuschreiben. Beide hat er an Blutungen verloren.

Herr Olshausen plaidirt für eine Behandiung ähnlich der bei Ovarialtumoren. Er hat vier Fälle mit Myomen dementspre- 
chend operirt. In zweien ist binnen wenigen Secunden Tod an Embolien der Lungenarterie eingetreten, was er nicht als zufällig erachten könne. Zwei seiner Fälle waren gestielte Myome; der dritte ein kleines Myom der hinteren Uteruswand, der vierte ein kaum gestieltes Myom. Für das beste Verfahren hält er die keilförmige Excision mit Zusammennähen. Er ist für vorläufige Behandlung des Stieles mit Draht und dann mit Seide. Bei einem breitbasigen Tumor hat er das Peritoneum umschnitten und dann den Tumor herausgeschält.

Herr Freund will den Stiel durch das hintere Laquear nach aussen fixirt und festgenäht haben.

Herr Martin operirte vier Fälle ganz nach Péan; alle vier starben.

\section{Sitzung vom 13. September 1878.}

Auf Vorschlag des Präsidium wird der Vortrag des Herrn Kocks auf diese Sitzung verlegt, um eine zusammenhängende Besprechung iiber das bremende Thema der Totalexstirpation des Uterus speciell ,des Freund'schen Verfahrens" anzuregen.

17) Herr Kocks (Bern): Demonstration eines exstirpirten Uterus und dreier dabei gefundenen Orarien.

Redner schickt der Schilderung seines Falles eine umfassende Geschichte der Uterusexstirpation voraus. Er selbst operirte, ohne Freund's Arbeit gekannt zu haben (im April 1878), im Wesentlichen nach der Methode von Sauter und Bundell. Er habe sich durch Injectionen der Arteria uterina, überhaupt durch das anatomische Studium der Lage dieser Arterie zum Uterus davon überzeugt, dass die Exstirpation des Uterus möglich sei, ohne die Uterina zu verletzen, indem das Messer dicht am Uterus in die Höhe gehen müsse. In seinem Falle handelte es sich um eine 39jährige Multipara mit Cervicalcarcinom ziemlich späten Stadiums. Operation unter Spray. Die Eingeweide nicht nach aussen gelegt, sondern mittels Handtuch nach oben gehalten. Uterus mit einer L üer'schen Zange gefasst, nicht durchstochen, um Austritt von Jauche aus dem Uterus zu verhüten. Die Blase wurde geschützt durch Einführen des Fingers nach vorheriger Erweiterung der Urethra durch einen Uterusdilatator und Vorschieben des Fingers.zwischen den Branchen. Die Ovarien wur'- 
den mitgenommen. Im Ligamentum latum sinistrum fand sich ein drittes Ovarium mit frisch geplatztem Graaf'schen Follikel. Das Peritoneum wurde von oben her mit fünf Nähten vereinigt. Patientin genas.

Zehn Tage nach der letzten Menstruation war operirt worden. In dem dritten Eierstocke fand sich ein Corpus luteum neben dem frischen Graaf'schen Follikel. (Demonstration des Präparates.)

18) Herr Veit: Einige Fälle von Krebs des Uteruskörpers.

Herr Veit demonstrirt drei von Schröder nach Freund exstirpirte carcinomatöse Uteri, und schliesst daran die Demonstration und Besprechung eines vierten Falles, den er selbst wegen Corpuscarcinom vor vierzehn Tagen, bisher mit Glück, operirt habe.

In der Discussion trat zuerst Herr Freund auf. Ein Bedürfniss nach der Operation sei entschieden vorhanden. Die Erkenntniss der Indication sei das Wesentliche. Und diese wurde zuerst von Hegar und Kaltenbach gemacht. Sie wiesen darauf hin, dass die Haupterfordernisse bei Totalexstirpationen des Uterus die seien, die Blutung zu stillen und eine gute Bedecknng zu schaffen. Diesen beiden Haupterfordernissen habe Freund durch anatomisches Studium und Anpassung an deren Ergebnisse zu genügen gesucht. $\mathrm{Er}$ habe bisher zehn Mal operirt, zwei Mal auswärts (Prag und Heidelberg), in beiden Fällen Tod durch septische Peritonitis; acht Mal in Breslau, wovon fünf genasen. Ein Fall ging an Sarkom des Darmes zu Grunde (zwischen Blase und Flexura). Bei der Section fand sich aber die Operationswunde geheilt. Im zweiten Falle trat am zwölften Tage Tod an Ileus ein. Der dritte Fall starb in Asphyxie. (Fettherz und Atrophie der Nieren.)

Darauf macht er folgende Verbesserungsvorschläge:

1) Der Hautschnitt ist bis in den Mons Veneris zu machen.

2) Bei jugendlicheren Personen Tenotomie der Musculi recti, um oberhalb der Symphyse einen dreieckigen Raum zu bekommen.

3) Das Peritoneum soll nicht bis in den untersten Wundwinkel gespalten, sondern an die Bauchwand angeheftet werden.

4) Die Därme können auch innerhalb der Bauchhöhle zurückbehalten werden.

5) Der Uterus soll nur dann durchstochen werden, wenn seine Höhle nicht carcinomatös jauchig ist. Er empfehle, den Uterus 
mit einer Art von breiter Klammerpolypenzange zu fassen, wobei man bis zu einem gewissen Grade den Uterus blutleer machen könne.

6) Was die Ligaturen der Ligamenta lata anginge, so hielten die oberen immer sicher. Am schwierigsten sei die dritte Ligatur. Eine Hufeisenligatur schliesse die Arteria uterina nicht völlig zusammen; daher legt Freund dieselbe doppelt an und bringt die Einstichkanäle dicht nebeneinander. Die von ihm construirte Troikartnadel erleichtere die Ligatureinlegung wesentlich.

Ehe Freund das Peritoneum vorn und hinten vom Uterus umschneidet, legt er Fäden ein, die er zur Naht des Peritoneum sogleich benutzt, so dass die Wundränder sofort vereint werden können. Nachdem der Uterus gegen die Blase hin abgetrennt worden, wird das Laquear von unten her mit gedecktem Messer durchstossen und durch Einführung zweier Finger von oben her in den Muttermund wird der letztere in die Bauchhöhle hineingedreht. Damit wird erzielt erstens eine grössere Leichtigkeit der Ausschneidung, zweitens wird vermieden, die angelegten Schlingen wieder zu durchschneiden, drittens die Unterbindung der kleinen Vaginalgefässe ermöglicht.

7) Für die Inversion der oberen Nähte ist ein starker Zug erforderlich; deshalb empfiehlt er Stäbchen aus Zink in die Suturen einzuschliessen, welche mit angezogen werden. Der Peritonealüberzug der Tuben und der Ligamenta ovarii wird in die Peritonealnaht mit aufgenommen, weil es in einem tödtlich abgelaufenen Falle passirte, dass die seitlichen Winkel nicht ganz geschlossen waren.

8) Bezüglich der Nachbehandlung empfiehlt er 2-3malige Ausspülungen der Vagina mit Carbolwasser pro die. Durch die Torsion des Uterus mit einem Theile des Laquear in die Bauchhöhle werde vermieden, dass Carcinommasse am Laquear unbemerkt sitzen bleibe, wie es sich in seinem Prager Falle bei der Section auswies, und wie es in dem von Fränkel in der Berliner klinischen Wochenschrift veröffentlichten Falle ebenfalls vorlag. In letzterem wurde bekanntlich der Rest von Carcinom mit dem. Paquelin'schen Brenner nachträglich abgetragen.

Fernerhin schlägt Freund vor, die Frage der Indicationen bis zum nächsten Jahre zu verschieben wegen der noch möglichen Recidive. Trotz scheinbarer Intactheit des Uterus habe sich bei den acht Breslauer Uteris stets das Corpus mit ergriffen gezeigt. Ein Mal habe sich im Fundus ein über erbsengrosses 
Carcinom gefunden, wo sich nur an der Portio ein kleines carcinomatöses Ulcus befand. Dieser unerwartete Umstand sei für die Indication sehr wichtig. Die Wirkung der Operation sei eine wohlthätige und sichere. Die durchgekommenen Operirten seien bis jetzt gesund geblieben.

Herr Schröder verfügt über drei Fälle von ,Freund'scher Operation", welcher Titel derselben allgemein zuerkannt werden sollte. Er hält die Operation für sehr schwer, aber für sicher ausführbar; und darum bei zwingender Indication für berechtigt. Die Därme liess er stets aussen. Die zwei oberen Ligaturen hat er in eine zusammengefasst. Ausserdem hat er die Ligaturen kurz abgeschnitten und dann durch das Peritoneum ein Diaphragma gebildet. Auch Recidive dürften nicht abschrecken, ebensowenig als sich die Chirurgen durch solche abschrecken liessen. Kämen auch einzelne Recidive vor, andere Fälle würden doch definitiv geheilt werden.

Er glaubt die Frage der Indication doch bereits jetzt berühren zu müssen. Die Operation sei angezeigt, erstens bei Fällen von Cervixcarcinom, welches öfters ganz versteckt auftrete. Die Vaginalportion sei scheinbar intact, in der Cervicalhöhle werde dann die Carcinommasse nachgewiesen. Das Carcinom gehe zuerst immer die Schleimhaut entlang in die Höhe in den Uterus hinein. Fälle, wo das Carcinom auf die Scheide übergehe, seien weniger geeignet. Hier sei ein anderes Verfahren einzuschlagen, indem man in sicherer Entfernung von der carcinomatösen Masse in das Laquear einschneide und den unteren Uterinabschnitt mit excidire, also supravaginale Excision des unteren Uterusabschnittes vornehme. (Vergl. Sitzungsberichte der Berliner geburtshülflichen Gesellschaft.)

Herr P. Müller hat zwei Fälle, allerdings nicht streng nach Freund, operirt. Einen Fall von Sarcoma uteri, Ligatur und Schlinge wurden unterhalb der. Neubildung angelegt. Genesung. Der zweite Fall war ein Carcinom des Corpus. Abtragung desselben mit Erhaltung der Vaginalportion, welche nicht carcinomatös war. Schlingenanlegung, sonst nach Freund. Die oberen Ligaturen wurden durch das Diaphragma der Vaginalportion nach unten und aussen geführt. Operation vor sechs Wochen. Bis jetzt kein Recidiv.

Herr A. Martin berichtet über zwei eigene Fälle und einen mit Dr. Langenbuch zusammen operirten. Misslich erschiene 
ihm die vollständige Eventeration. Deshalb habe er an der Leiche einen queren Halbmondschnitt mit dem Bogen nach unten versucht, aber die Muskelverletzungen seien zu ausgedehnt, und die Därme wurden unter der, durch den oberen Lappen gebildeten Schürze schwer zurückgehalten. Er wandte Freund's Instrumente nicht an, sondern durchstach das Laquear mit einer Péan'schen Nadel. Die Fäden schnitt Martin ebenfalls kurz ab. Bei der Operation sass er zwischen den Beinen der Frau. Eine starb an Sepsis, die andere an Collaps.

Herr Olshausen hält die Operation für sehr schwierig. Besonders sei es die Loslösung des Uterus und die Durchstossung des Laquear. Verletzungen der Blase und Ureteren seien schwer zu vermeiden. Er habe zwei Fälle operirt. Im ersten Falle erfolgte einige Tage lang Urinabgang durch die Scheide. Das Anfassen des carcinomatösen Uterus mit einer Klemmzange halte er für nicht gut wegen der Ausquetschung des Organes. Lieber möge eine Blutung erfolgen. Die Ovarien nahm Olshausen mit. Ein Fall ging an Blutverlust zu Grunde. Bei der Section ergaben sich die Lumbardrüsen als carcinomatös, und fand sich ein Carcinomknoten in der Niere. Im zweiten Falle trat Recidiv auf, und es sei dieser wohl der erste, wobei ein solches beobachtet worden sei. Am 28. Mai habe er operirt, und jetzt zeige sich ein Recidiv in der Vagina.

Herr Freund nahm im geschlechtsreifen Alter die Ovarien gesondert weg. Bei älteren Frauen habe er sie nicht entfernt. Gegen Martin sich wendend, bemerkt er, ohne zwingenden Grund sein Verfahren nicht ändern zu wollen, hauptsächlich wegen der Gefahr, Ureteren und Blase zu verletzen. Diese würden nicht verletzt, wenn man das Diverticulum uterovesicale frei lege und von unten her das Laquear durchtrenne. Nur wenn ein schrumpfender Process an der Basis des Ligamentum latum bestehe, könne der Ureter verzogen sein und verletzt, oder in die Schlinge eingeschlossen werden. Bei Carcinomen sei es auch mitunter bei vorheriger Untersuchung schwer zu sagen, ob im Parametrium blos Entzündung oder Neubildung vorliege. Treffe man altes parametritisches Narbengewebe an, so müsse man das Messer streng neben den Uterus halten; auch die Fixirung des Uterus sei schwer, weil derselbe öfters von ungemeiner Weichheit angetroffen werde; mit seiner Balkenzange brauche man durchaus nicht so stark zu comprimiren. Er stimme Olshausen im Allgemeinen bei und man müsse eben je nach den Verhältnissen verfahren. 
Herr Schröder findet es leicht, die Blase zu vermeiden. Habe man das Peritoneum zwischen Uterus und Blase durchtrennt, so könne diese wach vôrn gezogen werden. Die Durchstossung des Laquear könne auch von oben her ohne das Freund'sche gedeckte Messer geschehen. Auch ihm sei ein Fall von Infiltration des Parametrium vorgekommen, welcher zur Verwechselung mit Carcinom hätte Anlass geben können. Der Umstand, dass die Ovarien mit eingeschlossen waren, führte zur Verwerfung der Diagnose auf Carcinom. Daraus ergebe sich die Wichtigkeit einer vorherigen genauen combinirten Untersuchung mit Abtastung des kleinen Beckens.

Herr Baumgärtner (Baden-Baden) führte mit Hegar ein Mal die Operation aus. Carcinom der Portio; zuerst trichterförmige Excision. Ein halbes Jahr später Recidiv. Infiltration dos hinteren Laquear. Freund'sche Operation unter grossem Blutverluste, so dass man eine Anzahl Pincetten in der Scheide liegen lassen musste. Antiseptische Irrigationen. Tod am dritten Tage wegen Sepsis. Der Fall war ungeeignet.

19) Herr Dohrn: Demonstration von Hymenalpräparaten.

Die Bildung von Papillen und Falten erklärt sich aus dem Vorhandensein eines Gewebsüberschusses. Demselben Umstande verdankt an der Grenze der äusseren Haut auch die Anlage des Hymen seinen Ursprung. Der Hymen entsteht nicht an der Stelle des Sinus urogenitalis, sondern im untersten Abschnitte des aus den Mïller'schen Gängen hervorgehenden Vaginalschlauches. D ohrn weist dies an Sagittalschnitten von Föten, sowie an dem Präparate eines Fötus mit Vagina duplex, nach. Die Anlage des Hymen geschieht nach Dohrn erst in der 19. Woche des Embryonallebens. Bei Neugeborenen ist der Hymen nicht angespannt, sondern hängt schürzenartig heraus.

Herr v. H offma n n (Wiesbaden), welcher über dasselbe Thema noch nicht zum Abschluss gebrachte Untersuchungen angestellt hat, äussert sich theils beipfiichtend, theils in ausführlicher Weise über die von ihm gewonnenen Resultate. Dieselben werden später veröffentlicht werden, und gipfeln darin, dass der Hymen ein Nebenproduct bei der Bildung der Vagina sei, und ursprünglich entsprechend den früheren Einmündungen der Wolf'schen Gänge in den Sinus urogenitalis doppelt durchbohrt sei. Andere entwicke- 
der 51. Versammlung deutscher Naturforscher und Aerzte in Cassel. 475

lungsgeschichtliche Momente im Vortrage berühren nicht das Thema der Entstehung des Hymen, sondern die der Blase, Vagina und Ureteren.

Herr Dohrn weist darauf hin, dass Herr von Hoffmann seine Untersuchungen nur an einem Präparate gemacht habe, während er gegen 800 Fälle examinirte. Nach seiner Ansicht gehe die Vagina aus den Müller'schen Gängen hervor und glaube er nicht, dass die Hymenalanlage doppelt perforirt sei.

Herr F reund macht darauf aufmerksam, dass etwa $3 / 4 \mathrm{Cm}$. hinter der Urethralmündung, zwischen den Querfalten versteckt, sich constant bei jeder Frau eine halbmondförmige glatte Stelle finde, welche den Ort der Einsenkung der Wolf'schen Gänge in die Vagina bezeichne.

Herr Beigel (Wien) nimmt für sich die Priorität in Anspruch für den Nachweis der Persistenz der Wolf'schen Gänge in den Wandungen des Uterus.

Herr C. Ruge bezweifelt die Richtigkeit dieser Beobachtung.

Herr von Hoffmann wünscht seine Angaben nur als $\mathrm{Hy}-$ pothese aufgefasst zu sehen, welche sich aber an die Untersuchungen von Rathke anschlössen.

20) Herr Schwartz (Göttingen): Ueber Ovariotomie bei Kindern.

Ich erlaube mir Ihnen ein Eierstockscolloid vorzulegen, welches ich einem vierjährigen Kinde exstirpirt habe - eine Rarität also. Klinisch wahrnehmbare Ovarialtumoren sind ja vor dem Pubertätsalter überall nicht häufig, und wo sie vorkommen, sind es meist Dermoide, am allerseltensten proliferirende Kystome. Von letzteren konnte $01 \mathrm{sh}$ a usen nicht mehr als etwa vierzehn Fälle in der Literatur entdecken; unter diesen sind aber noch manche, denen der anatomische Beweis der colloiden Natur des Tumors fehlt. Von Ovariotomien bei Kindern, und zwar 2-12jährigen Mädchen, finden sich nur fünf verzeichnet; drei derselben hatten günstigen Erfolg.

Der von mir beobachtete Fall hat ausser der Seltenheit noch das besondere Interesse, dass sich das Eierstocksleiden mit einer Art von Menstruatio praecox verband. Wie nämlich die Anamnese ergab, hatten sich bei dem Kinde, dessen dicker Leib schon seit $11 / 2$ Jahren bemerkt, aber erst im letzten Vierteljahre rapide geArchiv f. Gynäkologie. Bd. XIII, Hft. 3. 
wachsen war, Genitalblutungen eingestellt, gering an Quantität und kurz von Dauer, aber in mehrmaligen, freilich etwas unregelmässigen Zwischenräumen sich wiederholend. Diese Blutungen als menstruale aufzufassen, durfte man um so mehr geneigt sein, weil die äusseren Genitalien, wenn auch noch nicht behaart, doch durch ihre ungewöhnliche Schwellung und Grössenentwickelung auffielen. Ueberdies erstreckte sich diese Hypertrophie, wie schon die Digitaluntersuchung per Rectum ergab und später der Augenschein bestätigte, auch auf den Uterus, welcher nichts von Neubildungen oder sonstiger Gewebserkrankung, wohl aber Dimensionen, wie bei einer Zwanzigjährigen, wahrnehmen liess. Befremdlich war es mir dagegen, den rechten Eierstock - der Tumor war ein linksseitiger - ganz normal d. h. kindlich und geschlechtsunreif zu finden. Inzwischen wird man doch wohl den Impuls der periodischen Blutungen in den Reizungen suchen müssen, die durch das rapide Wachsthum des kranken Eierstockes gesetzt wurden.

Was sonst noch zur Beobachtung kam, will ich möglichst kurz zusammenfassen.

Das seit Monaten bereits permanent bettlägerige Kind fieberte bei der Aufnahme lebhaft und anhaltend (Puls 120-130, Abendtemperatur $\left.39-40^{\circ}\right)$, und war dem entsprechend in seiner Ernährung sehr herabgekommen. Der trommelartig gespannte Leib zeigte den verhältnissmässig colossalen Umfang von $83 \mathrm{Cm}$., war äusserst schmerzhaft gegen Berührung und fluctuirte nur sehr undeutlich und in ganz kleinen Abschnitten. Zur Feststellung der Diagnose bedurfte es der Chloroformnarkose und ausser der Manualuntersuchung auch der Punction. Letztere lieferte nur knapp $100 \mathrm{Gm}$. einer Flüssigkeit, die freilich nichts besonders Charakteristisches hatte, doch aber der sonst begründeten Annahme eines rom Eierstocke ausgehenden Tumors nicht widersprach. Am dritten Tage nach der Punction führte ich die Ovariotomie aus, unter beständigem Carbolregen und mit allen sonstigen antiseptischen Cautelen, welche in diesem Falle abzuschwächen oder gar wegzulassen ich nicht wagen mochte, obwohl ich mir der Gefahren einer Carbolintoxication bei Kindern wohl bewusst war. Ueber den sonstigen Verlauf der Operation ist nicht viel zu sagen. Eine breite Netzadhäsion liess sich unschwer unterbinden und lösen; etwas störend war nur, dass der sehr derbwandige und durchweg parviloculäre Tumor sich durch den Troicart nicht verkleinern 
liess. Um ihn herauszubringen, musste ich nicht allein den Bauchschnitt bis hoch über den Nabel hinauf verlängern, sondern auch den Sack selbst spalten und im Innern aufbrechen - ein Verfahren, welches ich des Blutverlustes halber gern vermieden hätte, hier aber nur deshalb besonders erwähne, um das zerfetzte Aussehen des Präparates und den Anschein einer grösseren centralen Höhlung, die im Leben nicht vorhanden war, zu erklären. Der Stiel war kurz, sehr breit und gefässreich; ich unterband ihn mit drei kettenförmig ineinandergreifenden Catgutligaturen, versenkte ihn und legte sodann nach Reinigung und Schliessung der Bauchhöhle den Lister-Verband an. Nach der Operation fiel die Temperatur sofort auf die Norm, und abgesehen von einer leichten Carbolreizung, welche sich gleichzeitig mit einem geringen Blutabgange aus der Scheide rom zweiten bis vierten Tage einstellte, verlief die Heilung vollkommen glatt, wie das, beiläufig gesagt, mit Ausnahme einer alten bereits elf Mal punktirten und aus reinem Mitleid, so zu sagen in extremis, operirten Dame, bei allen 16 Lister'schen Ovariotomien, die ich seit November 1876 ausführte, der Fall gewesen ist.

Der Tumor wog im frischen Zustande trotz seiner theilweisen Zerstörung noch nahezu $4 \mathrm{Kil}$. Anatomisch charakterisirte sich derselbe als proliferirendes glanduläres Kystom, welches nur in der Kleinheit und wenig charakteristischen Gestalt der Zellen etwas Eigenthümliches besitzt. Um die Structurverhältnisse zur Anschauung zu bringen, habe ich ein paar mikroskopische Durchschnitte mitgebracht, welche ich, wie die histologische Untersuchung des Tumors überhaupt, unserem pathologischen Anatomen, Herrn Collegen Orth verdanke.

21) Herr Schwartz: Ueber Inversion des Uterus durch Neubildungen.

Was ich weiteres mitzutheilen habe, bezieht sich auf die Umstülpung des Uterus. Von der puerperalen Inversion kann ich nicht mitsprechen; ich habe solche noch niemals weder im frischen noch im veralteten Zustande gesehen. Nur über die seltenere, durch Neubildungen veranlasste Form der Umstülpung habe ich ein paar Erfahrungen zu machen Gelegenheit gehabt und will dieselben hier benutzen, um einen Gegenstand zur Erörterung zu bringen, der mir noch keineswegs allseitig abgeschlossen zu sein scheint.

Der erste Fall, der mir im Sommer 1876 zu Gesichte kam, 
betraf eine 61jährige, in steriler Ehe lebende Nullipara. Bei derselben hatte die stets reichliche Menstruation angeblich niemals cessirt, war aber um die Mitte der fünfziger Lebensjahre unregelmässig, dann profus geworden, und nach und nach in permanenten Blutabgang mit atypischen, bald mehr bald minder bedrohlichen Exacerbationen übergegangen. Seit etwa einem halben Jahre hatte Patientin zeitweise, und namentlich bei Gelegenheit stärkerer Blutungen, einen fleischigen Körper bis in und vor die Schamspalte vortreten gefühlt.

Diagnostisch war leicht festzustellen, dass es sich um Prolapsus des vollständig invertirten, bereits in seniler Involution begriffenen Uterus handelte. Auch der Cervicalkanal war mit invertirt; erst beim Zurückschieben des Uterus in die Scheide stellte sich ein seichter Muttermundsring wieder her. Die Ursache der Umstïlpnng lag zu Tage; ein reichlich wallnussgrosses, submucöses Myom sass gerade der Mitte des Fundus breitbasig auf. Ich trug dasselbe mit dem Messer ab, musste dabei aber sehr vorsichtig vorgehen, weil das Myom bis ganz nahe an die Serosa heranreichte. Die nicht eben sehr erhebliche Blutung ward durch Tamponade gestillt und mittels dieser zugleich die Rückstülpung erstrebt, jedoch nur theilweise, nur für den Bereich des Cervicalkanales bis zum Orificium internum erzielt. Der Fundus, an dem sich die Schnittfläche zu einer nabelförmig eingezogenen Narbe zusammengezogen hat, ist eben unterhalb des äusseren Muttermundes liegen geblieben. Alle wiederholt angestellten Versuche mit der Hand oder durch fortgesetzten Tamponadendruck die Rückstülpung zu vervollständigen, blieben erfolglos. Ich stand auch bald davon $a b$, weil sie überflüssig waren. Seit Entfernung des Myoms hat sich niemals wieder eine Spur von Blutung oder sonst irgend eine Beschwerde gezeigt; die Patientin erfreut sich auch heute noch des besten Wohlseins und völlig ungestörter Arbeitsfähigkeit.

Eine solche Unschädlichkeit veralteter Inversion ist ja öfters schon, selbst bei jüngeren und noch menstruirenden Frauen beobachtet worden. Im Uebrigen widerspricht diese meine Erfahrung der gangbaren Vorstellung, der zufolge mit Entfernung der Ursache, mit Abtragung der betreffenden Neubildung, die Rückstülpung gewöhnlich sofort von selbst oder unter leichter Nachhülfe erfolgen soll. Das scheint, wie eine Durchsicht der Casuistik lehrt, doch nur ausnahmsweise und nur dann der Fall sein, wenn. 
der spontan eingetretenen oder unabsichtlich durch operativen Zug. herbeigeführten Umstiulpung die Abtragung unmittelbar oder mindestens doch in der allernächsten Zeit folgt.

In ätiologischer Beziehung bestätigt sich hier, was von Andern bereits betont wurde. Zunächst die seiner Zeit von $\mathrm{Carl}$ Mayer eben so entschieden als irrig bestrittene Thatsache, dass auch der virginale Uterus unter Umständen durch Neubildungen invertirt werden kann, eine Thatsache, für welche in neuerer Zeit Spiegelberg und Martin Belege beibrachten. Sehr auffällig trat in dem mitgetheilten Falle an der Ursprungsstelle des Myoms auch die partielle Schwächung der Uteruswand hervor, in welcher wir wohl mit Scanzoni die Voraussetzung und Bedingung für die Entstehung dieser Art von Inversionen zu suchen haben.

Inzwischen ist gerade dies doch ein Moment, welches mir noch weiterer Prüfung bedürftig erscheint. Jedenfalls zeigt sich die partielle Atrophie nicht immer so deutlich, wie in dem erwähnten Falle. Sie werden sich hiervon überzengen können, wenn Sie den hier vorliegenden, ebenfalls invertirten und erst vor vier Wochen von mir amputirten Uterus in Augenschein nehmen und namentlich die Stelle genauer betrachten wollen, wo ein nachträglicher Schnitt die intraparietalen Myome gespalten hat.

Das Präparat stammt von einer 49jährigen Hebamme, welche einschliesslich eines dreimonatlichen Aborts vier Mal, zuletzt vor 19 Jahren geboren hat. Früher gesund und regelmässig, immer aber sehr reichlich menstruirt, hatte die Frau im November vorigen Jahres beim Heben einer schweren Last das Gefühl gehabt, als habe sich plötzlich etwas im Leibe gesenkt. Eine Blutung war nicht sogleich, sondern erst bei der nächstfolgenden Regel, und zwar im höchsten Grade abundant eingetreten, und hatte sich von da an, ohne jemals vollständig zu sistiren, bei jeder Menstruation bald mehr bald minder excessiv wiederholt. Von verschiedenen Aerzten behandelt, war das Leiden bald als Prolapsus, bald als Polyp gedeutet, von einigen aber richtig als Inversion erkannt. Pessarien, die man der Frau gab, hatte sie natürlich nicht vertragen, und ein Versuch, den vermeintlichen Polypen abzubinden, war wegen unerträglicher Schmerzen, Erbrechen, Ohnmachten u. s. w. sofort wieder aufgegeben. Seitdem hatte man die Patientin nur symptomatisch behandelt; permanent bettlägerig hatte sie es selbst gelernt durch Einspritzungen von verdünntem Liquor ferri die Blutungen einzuschränken. Freilich 
nur mit sehr bedingtem Erfolg. Im höchsten Grade anämisch und heruntergekommen, Abends auch nicht mehr ganz fieberfrei, ward Patientin Anfangs August dieses Jahres nach Göttingen transportirt.

Die Diagnose hatte in der Chloroformnarkose nicht die mindeste Schwierigkeit. Ich fand den Uterus bis auf schwache, nur vorn und hinten nicht aber seitlich fühlbare Reste der Muttermundslippen vollständig invertirt, an seiner rechten Seitenkante mit mehreren wallnuss - bis hühnereigrossen Myomen be- und durchsetzt, im Uebrigen so hypertrophisch, geschwollen und vergrössert, dass derselbe bis zum Introitus vaginae herabreichend Becken und Scheide wie ein mässiger Kindskopf ausfüllte. Wollte man die Frau nicht sterben lassen, so war augenscheinlich ein radicaler Eingriff sofort und dringend geboten; es fragte sich nur welcher, Ausschälung der Myome und Rückstülpung oder Amputation. Erstere schien mir verwerflich. Allerdings hätte sich mit Hülfe eines Drahtschnürers oder eines langen Gummidrains, den ich bei der Amputation des hypertrophischen Scheidentheiles schon öfter zu solchem Zwecke verwandt habe, eine vollkommene blutleere Ausschälung der Myome ausfibhren lassen, aber es waren deren mehrere da, dieselben isolirten sich nur sehr undeutlich, wie weit sie gegen die Sérosa vordrangen, war nicht auszumessen, jedenfalls wäre die Verwundung sehr gross ausgefallen, vor Allem aber, es blieb zweifelhaft, ob und wie rasch die Rückstülpung sich werde bewirken lassen. Gelang diese nicht oder nur unvollständig, so standen Nachblutungen und Jauchungen in Aussicht, und dieser Gefahr durfte die ohnehin schon sehr reducirte Kranke nicht mehr ausgesetzt werden. Deshalb und da die 49jährige Patientin des Uterus recht wohl entrathen konnte, entschloss ich mich zur Amputation. Ich habe dieselbe ausgeführt nach einem Plane, der sich theoretisch in mir, wie gewiss in vielen Anderen schon lange festgesetzt, seine praktische Probe aber bisher noch nicht bestanden hat. Ich meine die Sicherung des Amputationsstumpfes durch die Naht - ein Verfahren, welches sich, von gelegentlichen literarischen Andeutungen abgesehen, in der operativen Gynäkologie von Hegar und Kaltenbach ausfiuhrlich besprochen und allen übrigen Amputationsweisen gegenïber als einzig richtige Methode vorgeschlagen findet. Letzteres gewiss mit Recht. Die hauptsächliche Gefahr der Amputation liegt doch in der Rückstülpung des durchschnittenen und noch nicht peritonitisch verlötheten Inversionstrichters, und dieser Gefahr kann 
man in sicherer und unschädlicher Weise nur durch die Naht begegnen. Indessen habe ich die Naht etwas anders angelegt, als es Hegar und Kaltenbach vorzugsweise empfehlen. Ich habe die Suturen nicht in der Richtung von vorn nach hinten über den Amputationsstumpf geknüpt, und zwar deshalb nicht, weil ich fürchtete, die derartig angelegten Nähte möchten energischen Reinversionsbestrebungen des Stumpfes nicht widerstehen, zu früh locker werden oder ausreissen. Nachdem ich durch Vorziehen des Uterus den Halstheil desselben zugängig gemacht, habe ich diesen behandelt wie den Stiel eines Ovarialtumors, $d . h$. denselben in querer Richtung mit drei in einandergreifenden Drahtsuturen durchstochen und sofort nach Knüpfung derselben die Abtragung vorgenommen. Absichtlich, und um Gangrän des Stielrestes zu vermeiden, hatte ich die Ligaturen nicht allzufest, sondern nur so weit angezogen, als nöthig schien, um die Peritonealflächen des Inversionstrichters dicht aufeinander zu bringen. Dem entsprechend spritzten anch noch drei Arterien des Amputationsstumpfes und mussten isolirt unterbunden werden. Der central belegene peritoneale Spalt ward dann vorsichtshalber noch mit drei kleinen Suturen geschlossen.

Der weitere Verlauf hat nicht ganz meinen Erwartungen entsprochen. Die Heilung war trotz antiseptischer Nachbehandlung keine völlig fieber- und eiterungslose, und kam auch erst in der dritten Woche zum Schluss. Eine Reinversion ist nicht oder doch nur so weit erfolgt, dass schliesslich der äussere Muttermund nicht allein vorn und hinten, sondern auch seitlich als ein sehr seichter Ring gefühlt werden konnte. Innerhalb desselben liegt der in seiner Mitte leicht sternförmig eingezogene Uterusstumpf, seiner Form und seinem Volumen nach einer kurzen Vaginalportion ähnlich.

22) Herr Breisky (Prag): Ueber Haematometra bei hochliegender, breiter Scheidenatresie.

Er operirte zwei Fälle mittels einiger von ihm angegebenen neuen Instrumente. Der eine Fall betraf ein 1כjähriges, der andere ein 17 jähriges Mädchen. Beide mit der scheinbar als acquirirt zu bezeichnenden Form der Affection. Sein Verfahren besteht in Fixirung des Katheters durch den einen. Assistenten, Einführung des Fingers in das Rectum, von dem aus man den äusseren Muttermund durchfühlen könne, Seitens eines zweiten Assistenten. Breite Incision von der Scheide her, stumpfe Trennung 
des lockeren Gewebes bis zur Grenze des vermutheten oder deutlich gefühlten äusseren Muttermundes; breiter Einschnitt mit seinem neuen ,Troikartmesser “; sodann wird längs der Troikarthülse eine ,Zangenkanüle" vorgeschoben, zwischen deren auseinanderweichenden Branchen eine sogenannte „Pfeifenkanüle“ eingelegt wird, d. i. eine Canüle à double courant mit breiterem, winkelig abgeknickten, pfeifenkopfähnlichen Endtheil, welcher wie ein intrauterines Pessar liegen bleibt und zu antiseptischen Injetionen der möglichst breit geöffneten Höhle benutzt wird. Die Gefahr der Sepsis sei nämlich am meisten zu fürchten. Wegen Bildung von Narbengewebe sei Stenose nicht zu vermeiden.

Herr Winckel operirte zwei Fälle, das erste Mal, bei einem 15jährigen Mädchen, schob er den Troikart durch die Brücke in den Tumor hinein und erweiterte dann seitlich mit dem Messer. Er war genöthigt, die Erweiterung später zu wiederholen. In seinem zweiten Falle bestand Duplicität des Uterus mit querer Obliteration der Scheide. Tod nach der Punction an Collaps in Folge von Perforation der papierdünnen Tube (Haematosalpynx).

Herr Schwartz weist auf die Gefahr der Blutung und Wiederfüllung der Höhle hin. Ist mehr für kleine Oeffnung, weil der excessive dünne Uterus eine grössere activ nicht zu verschliessen vermöge.

Herr Breisky betont dem gegenüber das Moment der Hypertrophie des Uterus bei Haematometra. Breiter Einsehnitt sei besser, ausser bei grossen Tubensäcken. Tubenberstung ist aber oft secundär.

23) Herr Kollmann (Basel) demonstrirt zwei menschliche, elf bis vierzehn Tage alte Eier. Dieselben weisen einen bläschenförmigen, mit feiusten Zöttchen behängten Balg auf, ohne nachweisbare Embryonalanlage. Von Bedeutung sei die unverhältnissmässige Grösse der Eibläschen gegenüber solchen ron Thieren aus gleichem Alter, ferner das Vorhandensein von Zotten, also eines Chorion, wo es wegen Mangels der Enbryonalanlage noch nicht zur Bildung eines Amnion gekommen sein komnte.

\section{Freitag Nachmittag.}

Vorsitzender Herr Gus serow.

24) Herr Landau (Berlin) lässt Quellstifte von Tupelo circuliren. $\mathrm{Zu}$ beziehen von Tiemann \& Co., New-York. 
Herr Freund stellte sogleich eine Probe an, indem er einen mittelstarken Stift in gewöhnliches Wasser brachte. Nach etwa zehn Minuten war derselbe bis zum dreifachen Umfange aufgequollen, ohne dabei an elastischer Festigkeit zu verlieren.

25) Herr C. Ruge (Berlin) demonstrirt auf Objectgläser ausgebreitet zwei menschliche Eier, etwas älter als die von Kollmann. In beiden war eine deutliche Fmbryonalanlage vorhanden.

26) Herr Baumgärtner (Baden-Baden) demonstrirt ein Präparat von Pyo-Salpingitis, Object einer mitgünstigem Erfolge durchgeführten Castration.

Das Präparat erweise die Wichtigkeit einer der mannigfachen Hegar'schen Indicationen für die Entfernung der Ovarien, ,chronische Entzündungsprocesse der Leber, des Beckenbauchfelles und Parametriums, wenn auch nicht primär durch pathologische Processe im Ovarium entstanden, doch durch die Ovulation stets von Neuem angeregt und recidivirend"s.

Es wurde dasselbe einem 32jährigen Mädchen entnommen, dem Baumgärtner das rechte Ovarium mit Tube entfernte und zwar, was das Leiden der Kranken betrifft, mit einem so vollständigen Erfolge, dass er bestätigen könne, es gehöre diese Operation zu den allerdankbarsten. Die Patientin hatte zuvor nicht zu beschreibende Schmerzen, vorzugsweise im rechten Hypogastrium, aber auch über die ganze Unterbauchgegend; Untersuchungen ohne Narkose konnten nie vorgenommen werden, jede Verrichtung war von heftigen Schmerzen begleitet, die Menses eine fürchterliche Qual. Die Untersuchung in der Narkose ergab: normale Lage des Uterus - das linke Ovarium war drei Jahre früher als multiloculäre Cyste entfernt worden - rechtes Ovarium etwas intumescirt, längs desselben eine daumendicke Anschwellung, die bis zur Linea terminalis reicht und daselbst fixirt ist, so dass das Ovarium mit dieser Anschwellung kaum beweglich erscheint. Es habe sich somit um eine Geschwulst der Tube gehandelt, die sich bei der Operation als eitrige Salpingitis herausgestellt habe.

Ueber die Technik der Operation, die er in Beisein und unter Mithïlfe der Herren Hegar, Müller und Berton ausgeführt habe, verweist Baumgärtner auf Hegar's ,Castration der Frauen" and führt nur an, dass er das seitlich fixirte. Ovarium und Tube zuerst aus Adhäsionen losziehen musste, worauf es alsdann leicht in die Wunde der Linea alba zu bringen war und doppelseitig mit Seide unterbunden wurde. Zuvor war noch 
eine durch breite Verwachsung an der Tube adhärente Darmschlinge zu lösen.

Bei der seitlichen Fixation des Ovariums mit Tube an der Linea terminalis hatte Baumgärtner zuerst an den Flankenschnitt gedacht, zog aber den Schnitt in der Linea alba vor, um einen nach früherer Laparotomie entstandenen Bauchbruch durch die neue Naht wieder zu entfernen. In dem reichlich starken Ovarium sind eine Reihe Graaf'scher Follikel verschiedener Entwickelung zu sehen, eine rege Ovulation. Die Periode war meistens einige Tage vor der Zeit erschienen.

Der äussere Theil der Ala vespertilionis ist durch entzündliche Einlagerung verdickt, das Ovarium fest mit dem Trichter verwachsen, die Fimbrien desselben sind durch Entzündung untereinander und mit dem Ovarium verwachsen und verkümmert. Die Tube ist vom Morsus Diaboli bis zum Uterinalende fingerdick geschwollen und mit käsigem Eiter gefüllt, an dem äússeren Ende ausgebuchtete Stellen, die höchst wahrscheinlich in einiger Zeit zum Durchbruche gekommen wären.

Baumgärtner betont nun die Unmöglichkeit, in solchem Falle durch irgend welche Behandlung etwas leisten zu können. Die Patientin habe dreiviertel Jahre schwer geduldet und fortwährend starke Morphiuminjectionen gebrauchen müssen. Schon den Morgen nach der Operation habe sie einen völlig umgeänderten Gesichtsausdruck gehabt, dem der schmerzlichen Angst folgte der Ausdruck der Ruhe und Zuversicht.

Auch eine Zeit der Periode, die somit nicht erschienen war, ist seitdem ohne irgend welche Störung vorübergegangen.

Es ist dieser Fall, wie Baumgärtner erzählt, noch besonders durch die Anamnese interessant. Drei Jahre vor der Castration wurde die Patientin linksseitig ovariotomirt, und,zwar mit extraperitonealer Versorgung des Stieles unter ganz günstigem Verlaufe, so dass Patientin wieder schwere Arbeit versah. Fünfviertel Jahre später hatte Patientin plötzlich Stiche im Stiele und Blasengegend bekommen, weshalb Baumgärtner den Stiel aus seiner Bauchverbindung und seiner Verwachsung mit dem Scheitel und Grunde der Blase auslöste, worauf Patientin sich wieder gut erholte. Das rechte Ovarium und Tube sei damals völlig frei von pathologischen Veränderungen gewesen.

Baumgärtner glaubt die früheren Entzündungen und gemachten Laparotomien nicht als ursächliche Momente der eitrigen 
der 51. Versammlung deutscher Naturforscher und Aerzte in Cassel. 485

Salpingitis betrachten zu können, welche zur dritten Laparotomie beziehentlich Castration geführt habe.

Nähere, ihm nicht unwichtig erscheinende Details dieses Falles behält sich Baumg ärtner vor, bei anderweitiger Veröffentlichung zu geben, für heute kam es ihm darauf an, die Berechtigung und Wichtigkeit der von $\mathrm{Hegar}$ angegebenen Indication für Entfernung der Ovarien hervorzuheben.

Herr Schwartz interpellirt den Vortragenden auf die von ihm angewendete Stielbehandlung.

Herr Baumgärtner replicirt, dass er die extraperitoneale Stielversorgung vorziehe. Die Bauchhöhle werde von ihm ausgewaschen mit $30^{\circ}$ warmem Wasser. $\mathrm{Zu}$ diesem Behufe bediene or sich eines Dilatatoriums für das hintere Laquear, welches dem Lidhalter ähnlich gebaut sei. Zwischen die Branchen werde eine Drainageröhre eingeschoben. (Demonstration des Instrumentes.)

Herr Kocks knüpft an den in der Discussion berührten Punkt an über die Haltbarkeit des Catgut gegenüber der Seide, und berichtet über seine Experimente, welche er mit dem beiderseitigen Material an Laminariastiften, welche nachher in Aufquellung versetzt wurden, erhalten habe. Zuerst löste sich ein Seidenfaden, dann ein. Catgutfaden, und so fort fast in regelmässiger Abwechselung. Zum Mindesten stehen sich somit beide Stoffe gleich.

27) Herr Rothe (Altenburg): Demonstration eines Dammschutzlöffels.

Obschon der Dammriss selten zu den ernsteren Ereignissen zählt, ist er doch meist eine unangenehme Zugabe für Arzt und Hebamme sowohl, denen die Schuld zugeschoben wird, als für die Patientin. Das Hauptmittel zu seiner Verhütung, die Unterstützung des Dammes, ist unzuverlässig, auch nicht ganz ohne Bedenken, da nach Einigen gerade der Druck, welcher auf das ausgedehnte Perinäum zwischen Kopf und Hand ausgeübt wird, durch die, wenn auch nur kurz andauernde Ernährungsstörung dasselbe zum Zerreissen prädisponiren soll. Jedenfalls hat man im entscheidenden Augenblicke oft das Gefühl, als müsse man den Kopf, nicht den Damm unterstützen, um durch Erhebung des ersteren den letzteren zu entlasten, wenn nur das kleine, schlüpfrige, im Durchschneiden begriffene Segment einen Angriffspunkt gewährte.

Bei einer Zangenoperation, während welcher die Löffel we- 
gen zu starken Hängebauches vor vollständiger Entwickelung des Kopfes abgenommen werden mussten, drohte der in der Scheidenkrönung stehende Kopf den äusserst gespannten Damm jeden Augenblick zu sprengen. In dem eben erwähnten Gefühle führte ich den linken Löffel wieder ein, liess ihn durch sanfte Pendelbewegungen nach hïnten gleiten, bis er mit der Concavität nach vorn an der hinteren Commissur lag, übte einen sanften, gleichmässigen Druck nach oben aus, und im nächsten Augenblicke rollte die nächste Wehe den Kopf über die glatte, geölte Fläche des Löffels ohne Verletzung des Dammes.

Eine einfache Modification des Zangeulöffels ist nun dieser „Dammschutzlöffel“. Die Beckenkrümmung wurde da weggelassen, der kurze hölzerne Griff etwas mehr zurückgebogen, um zwischen den Schenkeln der Gebärenden gehalten andere Manipulationen nicht zu hindern, das Blatt schmäler und dünner gemacht und das Fenster beibehalten, um möglichst leicht und ohne Druck zwischen Kopf und Scheidenwand einzudringen.

Selbstverständlich kann der Löffel nicht jeden Riss verhüten, da er nicht allen Bedingungen, unter denen dieser entsteht, begegnet. Auch können wir nie mit Sicherheit behaupten, durch unsere Massnahmen einen Riss verhütet zu haben, weil uns der Beweis fehlt, dass er nicht auch ohne dieselben unterblieben wäre. Wohl aber können wir es mit annähernder Wahrscheinlichkeit vermuthen in den Fällen, wo es sich um Mehrgebärende handelt, die bei früheren Entbindungen Dammrisse erfuhren. In zwei solchen Fällen habe ich den Löffel mit dem erwünschten Erfolge angewendet, bei einer IIIpara, die bei schnell verlaufender Geburt zwei Mal ziemlich ausgedehnte Rupturen erlitten, und bei einer Ipara, die ich mit der Zange entband, während ich den zuletzt noch eingeführten Schutzlöffel der Hebamme zur Unterstützung des Kopfes übergab.

Die Einfachheit des Instrumentes und seiner Handhabung dürfte gestatten, dasselbe eventuell Hebammen anzuvertrauen.

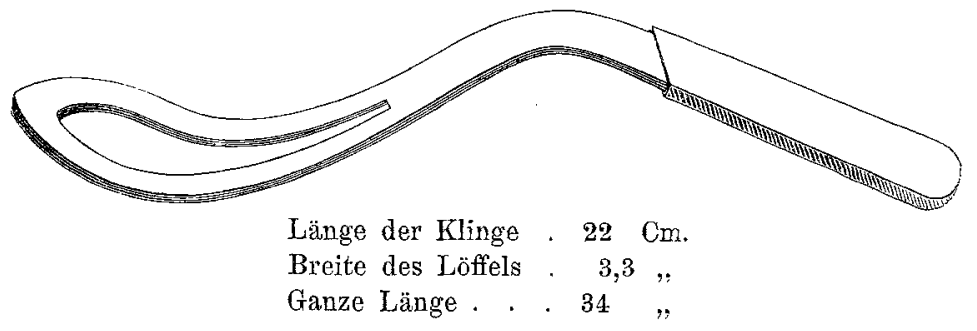


der 51. Versammlung deutscher Naturforscher und Aerzte in Cassel. 487

28) Herr Hofmeier (Berlin): Ueber Nephritis acuta gravidarum.

Redner weist auf die Häufigkeit von Nephritis in der Schwangerschaft hin. Ausser der Gefahr des Eintrittes von Eclampsie sei die Prognose gut. Im Wochenbette erfolge gewöhnlich die Heilung. Anders verhält es sich mit der chronischen Nephritis in der Gravidität und den Fällen von Uebergang aus der acuten in die chronische Form. Selten werde hier das Ende der Schwangerschaft erreicht. Man könne daher die Frage aufwerfen, ob nicht durch vorzeitige Unterbrechung der Schwangerschaft verhütet werde, dass eine acute Nephritis, welche in und durch die Schwangerschaft entstehe, einen chronischen Verlauf nehme. Bei gefahrdrohenden Symptomen plaidirt Redner selbst für künstlichen Abort.

Herr Olshausen weist noch besonders auf den Zusammenhang der Nephritis mit der Schwangerschaft hin. Namentlich für die Uebergangsformen aus acuter in chronische Nephritis sei die Prognose schlimm. Redner stimmt dem Vortragenden bei mit Ausnahme des Punktes, dass acute Nephritis, verbunden mit Eclampsie, wenn die letztere auch vorübergehe, prognostisch so günstig sei. Zudem sei noch gar nicht erwiesén, ob bei Eclampsie auch stets Nephritis vorhanden sei. Es handle sich hier meist um acute Nierenstauung mit Staungsalbuminurie, nicht um Nephritis. Wo eine solche unzweifelhaft besteht, gerade da tritt keine Eclampsie auf. Einmal sah Olshausen an einer Frau zwei Mal Eclampsie auftreten, wobei sich herausstellte, dass sie an Nierenschrumpfung, nicht aber an parenchymatöser Nephritis litt.

29) Herr Hirschberg (abwesend) lässt ein Hysterotom circuliren.

30) Herr P. Müller (Bern) regt aufs Neue eine Discussion an über die noch immer nicht gelöste und streitige Frage vom Verhalten der Cervix in der Schwangerschaft.

$\mathrm{K}$ üstner wolle neuerdings, wie in ähnlicher Weise früher Bandl, nachgewiesen haben, dass die Cervicalschleimhaut sich in eine Decidua umwandle. Müller tritt dem ganz entschieden entgegen. Fritsch hat darauf hingewiesen, dass Redners Fälle (durch Congelation gewonnene Präparate) unrichtige Befunde dargeboten hätten. Darauf hin habe Redner einen nächsten Fall 
in einer anderen Weise behandelt, nämlich mittels Härtung in Chromsäure und Alkohol. Die Durchschneidung des Uterus ergab, dass die Cervix ganz erhalten war; mikroskopische Untersuchung der Cervicalschleimhaut habe er ebenfalls vornehmen lassen und zwar durch Langhans. Die Bandl'sche Ansicht, dass die Cervix sich bereits in den ersten zwei Dritteln der Schwangerschaft erweitere, sei entschieden unrichtig. Wo es der Fall sei, lägen Geburtserscheinungen vor.

Herr Ruge: Er habe schon früher den anatomischen Weg für die Untersuchung der vorliegenden Frage eingeschlagen. Man hätte sich vor Allem zu fragen, wo hört der Cervicalkanal auf, und wo beginnt Decidua. Wo letztere sei, müsse man das Corpus uteri vor sich haben, wo nicht, die Cervix. Ebell (Verhandlungen der Berliner geburtshülflichen Gesellschaft) habe den Bandl'schen Muttermund als den mechanischen bezeichnet. Darüber liesse sich verständigen. Bei Molenschwangerschaft, wo binnen kurzer Zeit doch eine sehr bedeutende Ausdehnung des Uterus eintrete, ganz wie in späteren Monaten einer normalen Schwangerschaft, fände sich kein Verstreichen der Cervix wie bei späterer Zeit der Schwangerschaft. Der innere Muttermund sei geblieben. Auch könne man an jedem puerperalen Uterus ein bis vier Stunden post partum gute Beobachtungen anstellen, wie der Bandl'sche, resp. mechanische Muttermund allmälig tiefer trete bis zum Zusammenfallen mit dem inneren Muttermunde von P. M ïlle r. Redner hofft auf eine baldige Erledigung der Streitfrage.

Herr Leopold weist ebenfalls darauf hin, dass der innere Muttermund mikroskopisch-anatomisch festzustellen sei. Er habe Präparate untersucht aus der 30., 36. bis 40. Woche von Erstgeschwängerten, bei denen sich der $5-6 \mathrm{Cm}$. lange Cervicalkanal fest geschlossen fand, und wo der anatomische innere Muttermund genau auf den klinischen fiel. Der erstere war fest geschlossen. Bis zu ihm erstreckte sich innerhalb der Cervicalhöhle nur Cervicalschleimhaut, während sofort an ihm von der Umknickungsstelle an Decidua vera begann.

Herr Breisky ist für die klinische Beobachtung, da die anatomische noch nicht gelöst sei. Auskunft darüber erhalte man auch durch die künstliche Frühgeburt. Es sind Wehenerscheinungen, wenn der innere Muttermund auseinandergezogen wird. Ausserdem besteht alsdann Auflockerung, Dehnung, Verlängerung im Collum stets als Geburtserscheinung; stets ist die Geburt langsam im Gange. 
Vorwehen können zu jeder Zeit der Schwangerschaft auftreten, und damit ist auch die Möglichkeit gegeben, dass die Cervix, wie Bandl will, frühzeitig in den Uterus einbezogen werde. Bandl gab einfach eine Erläuterung für diese Vorgänge. Was das Verhalten der Cervix unmittelbar post partum angehe, so sei nur ein einziger Muttermund als Wulst zu fühlen.

Herr Müller: Die klinische Seite der Frage sei seiner Zeit schon in Hamburg erörtert worden. Nicht blos am Ende, sondern schon am Anfange der Schwangerschaft habe Bandl damals gesagt, sei die Cervix in den Uterus zum Theil mit aufgegangen. Das wolle er als unrichtig hinstellen.

Herr $\mathrm{Ruge}$ ist mit $\mathrm{M} \ddot{u} l l e r$ für Auseinanderhaltung eines klinischen (mechanischen Bandl'schen) und eines anatomischen (Müller'schen) Muttermundes.

Herr Ebell begiebt sich mit Bre isky auf die klinische Seite der Frage. Durch combinirte Untersuchung könne man zuweilen den inneren Muttermund feststellen. Die Cervix habe bis zum inneren Muttermunde eine Länge von 1-4 Cm.

Herr Frits ch vertheidigt kurz seine früher geäusserte Ansicht.

Herr Schröder meint, nach seiner Auffassung sei zwischen dem anatomischen Muttermunde nach $M$ üller und dem Bandl'schen in der Mitte der wahre innere Muttermund zu suchen, als die Grenze zwischen Cervix und dem ausgedehnten Uterus.

Der Vorsitzende schliesst die Sitzungen der Section unter Hinweis auf die erspriessliche und rührige Thätigkeit der Sectionsmitglieder, denen er seinen Dank ausspricht.

Mit der Vorbereitung zu den nächstjährigen Sectionssitzungen wurden Herr Freund (Broslau) und Herr B. Schultze (Jena) betraut.

Dr. Leopold. Dr. Sänger. 